\title{
A Robust Localization System for Multi-Robot Formations Based on an Extension of a Gaussian Mixture Probability Hypothesis Density Filter
}

\author{
Alicja Wasik · Pedro U. Lima · Alcherio Martinoli
}

Received: date / Accepted: date

\begin{abstract}
This paper presents a strategy for providing reliable state estimates that allow a group of robots to realize a formation even when communication fails and the tracking data alone is insufficient for maintaining a stable formation. Furthermore, the tracking information does not provide the identity of the robot, therefore a simple fusion of tracking and communications data is not possible. We extend a Gaussian Mixture Probability Hypothesis Density filter to incorporate, firstly, absolute poses exchanged by the robots, and secondly, the geometry of the desired formation. Our method of combining communicated data, information about the formation and sensory detections is capable of maintaining the state estimates even when long-duration occlusions occur, and improves awareness of the situation when the communication is sporadic or suffers from shortterm outage. The proposed method is validated using a highfidelity simulator in scenarios with a formation of up to five robots. The results show that the proposed tracking strategy allows for sustaining formations in cluttered environments,
\end{abstract}

Supported by ISR/LARSyS Strategic Funds from FCT projects FCT[UID/EEA/5009/2013] and FCT/11145/12/12/2014/S.

A. Wasik

Distributed Intelligent Systems and Algorithms, EPFL, 1015 Lausanne, Switzerland

Tel.: +41216931213

E-mail: alicja.wasik@epfl.ch

P. U. Lima

Institute for Systems and Robotics, Instituto Superior Técnico, Av. Rovisco Pais 1, 1049-001 Lisboa, Portugal

Tel.: +351218418274

E-mail: pedro.lima@tecnico.ulisboa.pt

A. Martinoli

Distributed Intelligent Systems and Algorithms, EPFL, 1015 Lausanne, Switzerland

Tel.: +41216936891

E-mail: alcherio.martinoli@epfl.ch with high measurement uncertainty and low quality communication.

Keywords Multi-Robot Tracking - Formation Control . Cooperative Positioning System · Probability Hypothesis Density Filter · Cooperative Localization

\section{Introduction}

With the increasing interest in robotics, multi-robot approaches are providing solutions in a large variety of applications, ranging from industrial automation (Alonso-Mora et al 2015) and building inspection (Saska et al 2017), to cinematography (Nägeli et al 2017) and social applications (Wasik et al 2017b). As the robots are becoming more present in environments populated with humans, safety, robustness and reliability of the methods are of highest importance. While many of the multi-robot approaches do not require continuous coordination among agents, methods such as formation control and other consensus-based algorithms heavily depend on the state of all the robots, and the interaction involves constant information exchange between an agent and all of its neighbors in the network (Olfati-Saber et al 2007). For this reason, consensus-based methods require robust and reliable information flow among robots.

One of the most popular strategies for formation control is the leader-follower method, where a leader agent defines the team objective, guiding the formation through the environment, while the followers maintain desirable distances from the leader and from the other followers. Therefore, to keep a desired formation geometry, it is necessary that each robot maintains a good estimate of the poses of the other robots, called the formation state. A role of the robot in the formation is usually associated to the robot's unique identification (ID), not only to distinguish which robot is to be the leader, but also where a particular robot should be located 
within the formation as a follower. Therefore the robots must be capable of distinguishing each other.

To obtain the states and the IDs, the simplest solution for the robots is to communicate the required information to each other. Unfortunately, wireless communications suffers from many problems, including message losses, delays, and even temporal loss of connections. Volatile communications threatens formation stability, while temporary loss of communication during close proximity navigation might even lead to collisions. While communication is often the only possible approach to formation control in dynamic and structured environments full of obstacles (Wasik et al 2016a), it is necessary to provide a backup solution for securing the formation when the communication fails. An example of such situation is shown in Figure 1, where a formation of two robots breaks temporarily due to faulty communication.

Our goal is to maintain a robot formation in settings which are by far not standard for the majority of the formation methods, namely GNSS-denied, complex indoor environments populated with humans and obstacles, where a positioning system based on direct inter-robot measurement suffers from long-term occlusions and false detections (Wasik et al 2016a). We have worked with ID-based robot formations, where each robot is assigned a role in the formation, but laser-based sensory detection, proposed in Wasik et al (2016b), does not provide robot IDs. It is important that the desired formation geometry is maintained, but since the robots are homogeneous, they can assume any role (target position). Roles are important to determine the range and the bearing that the robot has to keep with respect to the other robots in the formation. Lack of ID information associated with the measurements does not allow for simple fusion of communication and tracking, especially when tracking is expected to sustain the formation dependably for some time, even when communication fails. Methods without explicit data association, such as PHD filters, require on-line role assignment, where the robot dynamically estimates the optimal matching of the estimates with the roles (including its own role that might change over time). When the assignment is not shared, the robots might diverge to different guesses of the assignments, making the formation ill-defined and leading to breaking of the formation. Wrong data association can be caused by as little as one track missing, where detection is not perfect and has several long term occlusions when the formation is moving while following a path. This challenge makes the tracking-only methods unsuitable for maintaining the entire formation for long periods of time.

To overcome such difficulties, we have developed a system that incorporates communication data, tracking information, and knowledge about the desired formation geometry in the Gaussian Mixture Probability Hypothesis Density (GM-PHD) filter (Vo and Ma 2006). The use of such filter
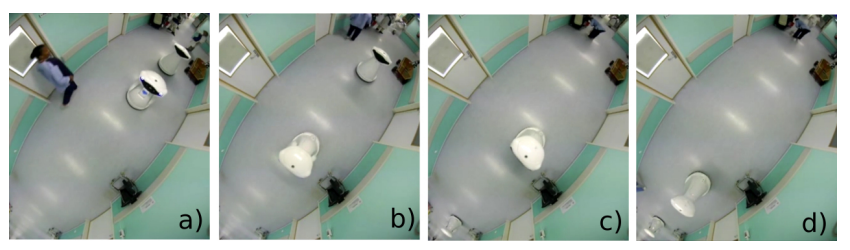

Fig. 1: Images from overhead cameras showing a formation of two robots navigating in a hospital environment (a). During execution, the robots change access point (b), which causes the formation to break temporarily (c) before it gets corrected after the robots communicate again (d). Similar problems could be solved if the robots used a combination of communication and tracking based on onboard sensing.

allows us to combine data from multiple information sources without the need to use heuristic methods for data association. Moreover, a GM-PHD filter does not set the number of tracks a priori, therefore additional data regarding a target can be incorporated seamlessly (Vasic and Martinoli 2015). Introduced in Wasik et al (2017a), our filter called Formation Information GM-PHD (FI-GM-PHD), to the best of our knowledge, is the first approach attempting to improve tracking estimates of the robot poses based on specification of the desired formation geometry. Our method consists of two main components added to the GM-PHD filter: i) the inception step incorporates poses of the robots exchanged via communication, when such information is available, ii) the coalition step integrates the projection of the formation state based on the desired formation geometry. The projected formation state is either improving the current estimate or generating a new one, depending on the dissimilarity between the estimated formation state and the projected formation state.

An abbreviated conference version of this paper has been presented in Wasik et al (2017a). The novelty of this work compared to Wasik et al (2017a) is fourfold. First, we lift an assumption that the formation always remains close to the desired geometry, by modifying the coalition step, where novelty now depends on the estimation of the performance of coalition matching, so that the closer the robots are to the perfect formation, the more likely a strong novelty is created for a temporarily untracked robot. Second, we introduce an improvement in the inception step, which is now performed as a PHD update. Third, the formation has a real robot leader instead of a virtual leader, therefore the pose of the leader no longer has to be broadcasted to all the robots. Fourth, we added a sensor model to determine the probability of detection.

The final goal of this work is to provide reliable robot pose estimates to be used when communications throughput is low or when communications fails. For safety reasons, such backup system is necessary for establishing co- 
operative multi-robot navigation in human-populated environments Wasik et al (2016a) (such as depicted in Figure 1), which is the long-term goal of this work.

The rest of this paper is organized as follows. Section 2 presents an overview of related work. Section 3 provides the background on multi-target methods and the GM-PHD filter. Section 4 states formally the problem while Section 5 describes the FI-GM-PHD filter. Section 6 explains how the estimates obtained from the FI-GM-PHD are used in the formation control algorithm. Experiments performed in a highfidelity simulator are introduced in Section 7, with the scenarios presented in Section 8 and the results presented in Section 9. The paper is concluded in Section 10.

\section{Related Work}

The objective of multi-target tracking is to jointly estimate the states of multiple targets, and sometimes the numbers of targets themselves, from a sequence of noisy observation sets. Existence of multiple targets and multiple measurements necessitates data association, a computationally expensive procedure dealt with either explicitly or implicitly. Therefore single-target tracking approaches are not readily extensible to the multi-target problems. From among many existing multi-target tracking algorithms that emerged in recent years, Qiu et al (2015) classified the existing approaches in the following categories: a) Non-Bayesian Approaches, such as Nearest Neighbor (NN) data association, b) Maximum A Posteriori approaches, such as Multiple Hypothesis Tracking (MHT), c) Bayesian estimators, such as Joint Probabilistic Data Association (JPDA) filter or Markov Chain Monte Carlo data association (MCMCDA), and $d$ ) $\mathrm{Fi}$ nite Set Statistics (FISST)-based approaches, such as Random Finite Sets (RFSs).

The NN methods deal with data association by assigning each measurement to the closest target based on a distance measure (Bar-Shalom and Fortmann 1988). The NN filters assume one-to-one mapping between the measurements and the targets, therefore cannot deal with multiple observations of a single object, clutter nor occluded objects. The JPDA filter is a sub-optimal Bayesian algorithm that calculates a marginalized probability on the joint data association space. To mitigate the computational burden, many of the heuristic techniques to an approximate JPDA sacrifice the tracking accuracy to make the algorithm computationally tractable and, as a result, the application domain is restricted to scenarios with few, well separated targets (Bar-Shalom 1990). MHT evaluates the likelihood that there is a target given a sequence of measurements. To restrict the exponential growth of the number of hypotheses, MHT requires pruning out spurious hypotheses for each track independently and discarding the deleted items, which makes it impossible for MHT to recover from errors (Blackman and Popoli
1999). The Probabilistic MHT (PMHT) uses soft association methods, but assumes that the number of targets is known, and that it is possible to initialize the states (Streit and Luginbuhl 1994). MCMCDA filter is a true approximation for the optimal Bayesian filter. However, the algorithm requires specification of numerous parameters, the target creation is accomplished heuristically and many particles are required for the method to perform well (Oh et al 2009).

The Random Finite Set (RFS) approach to multi-target tracking is a novel and promising alternative to the traditional association-based methods. RFS is theoretically optimal approach to multi-target tracking and a direct generalization of the single-target Bayes filter. Its main advantage is that it treats the problem of clutter and association uncertainty under a rigorous unified Bayesian filtering framework Mahler (2003). Moreover, it incorporates track initiation, a procedure that has mostly been performed separately in traditional tracking algorithms. Based on the RFS theory, the Probability Hypothesis Density (PHD) filter and its variations deal with the measurement-to-track association implicitly, resulting in higher robustness and accuracy in scenarios where the number of targets is not known in advance or changes over time.

Flexibility and ability to deal with challenging scenarios makes the PHD filters and the variations thereof increasingly popular in the robotics research. Among many other notable examples, the PHD filter is used for Simultaneous Localization and Mapping (SLAM) in Adams et al (2014), where it estimates vehicles trajectories and the encountered environment features, also in the multi-robot case in Moratuwage et al (2012). In Vasic and Martinoli (2015), collaborative tracking of multiple vehicles is achieved by fusion of PHD hypotheses among the collaborative agents. Cooperative multi-target tracking with the PHD filter is also exploited in Dames and Kumar (2013). The output of the PHD filter in Dames (2017) is used not only for tracking of targets, but it also provides importance weighting so that the robots are drawn towards areas that are more likely to contain targets. Sapkota et al (2016) perform visual detection and tracking with GM-PHD filter to estimate the aircraft's position and velocity in three dimensions. Melo and Matos (2014) track multiple autonomous underwater vehicles using acoustic signals and a Monte Carlo PHD filter. Stegagno et al (2013) is an example of application-driven modification of a PHD filter, where the identities of the unmanned ground vehicles are reconstructed by incorporating odometric data collected by an aircraft in the PHD filter.

The problem of tracking for realization of multi-robot formations has been addressed as early as the first approaches to formation control appeared. Among many existing formation control algorithms, the most common ones rely on the pose estimates in the global reference frame (Lewis and Tan 1997), on relative positions of the other 
robots (Falconi et al 2010), on range only (Cao et al 2011) or bearing only information (Bishop 2011). While acquisition of accurate state of the other team members has been addressed previously using communications as well as various perception tools, including cameras (Monteiro and Bicho 2010), infrared sensors (Falconi et al 2010), sonars (Soares et al 2013) or laser range finders (Wasik et al 2016b), tracking is significantly simplified in order to be reliable enough for formation control. Simplification usually casts a multitarget tracking problem to single-robot tracking by providing the robots with unique identifications (IDs) that can be extracted by the tracking robot. Using the estimates with known IDs is trivial, as long as not all the tracks are not lost.

To realize an ID-based formation, multiple solutions have been proposed in the literature. In Fredslund and Matarić (2002), robots perform teammate detection using combination of a LIDAR and a camera, where the camera also provides the identity of the neighboring robot. In Alur et al (2001), robots recognize themselves by extracting color blobs from a camera image. Both approaches aim at localizing a single local leader distinguishable by a marker. In Soares et al (2013), a follower vehicle maintains a formation with two leaders using acoustic ranging. The distinction between the leaders is ensured by an appropriate timemultiplexing scheme of the acoustic relative positioning signals. For tracking multiple quadrotors, Dias et al (2016) use active markers and an on-board camera. The markers provide $3 \mathrm{D}$ poses of the robots, and by pulsating at a predefined frequency, provide a unique aircraft ID. ID-dependent graph-based formation is achieved in Falconi et al (2010). Robots use a dedicated infrared range-and-bearing system and exchange messages containing robot IDs. In Ye et al (2017), agents in a formation localize using bearing-only measurements but are constrained to move with the motion type that is known by all agents a priori.

Within the context of multi-robot coordination, the problem of role assignment has been addressed previously using potential fields Zavlanos and Pappas (2008), market-based algorithm Michael et al (2008) for task allocation, and the Hungarian algorithm in Sun and Xia (2014) and in Zhang et al (2013) for formation initialization. It was also applied with graph-based formations (Falconi et al 2010). Those works however consider only a static case, i.e. the roles must be assigned only once, and are kept throughout the experiment. Dynamic role assignment, where roles are updated online by the robots as they navigate in the environment, can be performed as part of the tracking algorithm. Panta et al (2009) associate the state estimates within a GM-PHD filter with unique track labels. In Stegagno et al (2013) the IDs of the robots are reconstructed by incorporating odometric data directly in the PHD filter. Both methods maintains the tracklabel association, however assignment of the labels with for- mation roles would require further manipulation. Franchi et al (2013) tackle a problem of mutual multi-robot localization with ID-less measurements, where robots are allowed to communicate their IDs and the ID-less measurements of the other robots. The robots self-localize in their individual coordinate frames, the relative configuration of which has to be estimated. The proposed method uses probabilistic data association techniques, combinatorial in their nature, together with multiple particle filters, one per each robot.

Cooperative object tracking, studied in Ahmad et al (2017), which aims to localize a target of common interest, and cooperative prediction of environmental boundaries, studied in Saldaña et al (2017), are, in some sense, similar to the problem of tracking the robots in the formation. The objective of the above localization problems is to estimate the coordinates of one or more targets or locations based on available local information. The difference is that the localization problem studied here aims to localize all moving neighbors by each agent, while the cooperative tracking problems studied in Ahmad et al (2017) and Saldaña et al (2017) aim to localize a stationary or almost-stationary target by each agent.

Up to now, robot localization and multi-agent formation control are addressed as two separate problems in most of the existing research. There exist very few works which combine them as an integrated control problem. Jiang et al (2017) study velocity and relative position estimation integrated with formation control, but each agent is forced to carry out a combined circular and linear motion during the entire process. Cao et al (2011) devise a method called stop-and-go, to control the agents in the formation that are not able to measure the relative positions of their neighbors. This measurement restriction makes the control problem significantly harder. Han et al (2018) develop a consensus-like relative localization using measurements and local communications and integrate it with leader-follower formation control by combining the proposed relative localization scheme and a Laplacian-based formation control method. ID data is available to the agents as they communicate locally. The above methods mostly focus on the theoretical aspects of the problem and show only results in simulations with simplistic models. Assumptions on robot motion and availability of additional information make them not suitable for ID-based formations with ID-less tracking data.

\section{Background}

\subsection{Multi-Target Tracking using Random Finite Sets}

The Random Finite Sets (RFSs) are natural representations of multi-target states and observations that provide a way to directly extend single-sensor, single-target Bayes statistics 
to multi-sensor, multi-target problems (Mahler 2003). The RFS formulation treats the collection of individual targets as a set-valued state, and the collection of individual observations as a set-valued observation.

Let $M(k)$ be the number of the targets at time $k$ with the states $\mathbf{x}_{k, 1}, \ldots, \mathbf{x}_{k, M(k)} \in \mathcal{X}$. At the next time step some targets may die, surviving targets evolve to the updated states and new targets may appear. Let $N(k)$ be the number of measurements $\mathbf{z}_{k, 1}, \ldots, \mathbf{z}_{k, N(k)} \in \mathcal{Z}$. Measurements can be generated by the targets or stem from the clutter (false positives). Missed detections can occur due to sensing imperfections (false negatives).

At time $k$, the collections of target states and the collections of measurements can be represented as finite sets:

$X_{k}=\left\{\mathbf{x}_{k, 1}, \ldots, \mathbf{x}_{k, M(k)}\right\} \in \mathcal{F}(\mathcal{X})$

$Z_{k}=\left\{\mathbf{z}_{k, 1}, \ldots, \mathbf{z}_{k, N(k)}\right\} \in \mathcal{F}(\mathcal{Z})$

where $\mathcal{F}(\mathcal{X})$ and $\mathcal{F}(\mathcal{Z})$ are the collections of all finite subsets of targets $\mathcal{X}$ and measurements $\mathcal{Z}$ respectively ${ }^{1}$.

\subsubsection{The RFS State Evolution Model}

An RFS model for the time evolution of the multi-target state can incorporate target motion, birth and death of a target (Vo and Ma 2006). The targets may be temporarily occluded or venture out of the field of view. Thus, given a multi-target state $X_{k-1}$ at time $k-1$, the multi-target state $X_{k}$ at time $k$ is given by the union of the surviving targets and the spontaneous births:

$X_{k}=\left[\bigcup_{\zeta \in X_{k-1}} S_{k \mid k-1}(\zeta)\right] \cup \Gamma_{k}$

$S_{k \mid k-1}(\zeta)$ is the model of the behavior of the state $\zeta$ at the next time step and can take on either $\left\{\mathbf{x}_{k}\right\}$ when the target survives or $\emptyset$ when the target dies, corresponding to the cases where the target $\mathbf{x}_{k-1} \in X_{k-1}$ continues to exist at time $k$ with probability $p_{S, k}\left(\mathbf{x}_{k-1}\right)$ or dies with probability $1-p_{S, k}\left(\mathbf{x}_{k-1}\right)$ respectively. Targets that continue to exist transition from a state $\mathbf{x}_{k-1}$ to $\mathbf{x}_{k}$ with the multi-target transition density $f_{k \mid k-1}\left(\mathbf{x}_{k} \mid \mathbf{x}_{k-1}\right) . \Gamma_{k}$ is the RFS of spontaneous birth at time $k$.

\subsubsection{The RFS Measurement Model}

The RFS measurement model accounts for the uncertainty of detection and the clutter. Given a multi-target state $X_{k}$ at time $k$, the multi-target measurement $Z_{k}$ is given by the

\footnotetext{
1 Analogously to the single-target system, where uncertainty is expressed by modeling the state $\mathbf{x}_{k}$ and the measurement $\mathbf{z}_{k}$ as random vectors, the uncertainty of the multi-target system is expressed by modelling the multi-target state $X_{k}$ and the multi-target measurement $Z_{k}$ as RFS.
}

union of the measurements generated by the target and the clutter:

$Z_{k}=\left[\bigcup_{\mathbf{x} \in X_{k}} \Theta_{k}\left(\mathbf{x}_{k}\right)\right] \cup K_{k}$

where $\Theta_{k}\left(\mathbf{x}_{k}\right)$ is an RFS generated by the target with a state $\mathbf{x}_{k} \in X_{k}$ at time $k$ and can take on either $\left\{\mathbf{z}_{k}\right\}$ when the target is detected with probability $p_{D, k}\left(\mathbf{x}_{k-1}\right)$ or $\emptyset$ when the target is not detected with probability $1-p_{D, k}\left(\mathbf{x}_{k-1}\right)$. For the detected targets, the probability density of obtaining an observation $\mathbf{z}_{k}$ from $\mathbf{x}_{k}$ is given by the multi-target likelihood $g_{k}\left(\mathbf{z}_{k} \mid \mathbf{x}_{k}\right) . K_{k}$ denotes the clutter.

\subsubsection{The Optimal Multi-Target Bayes Filter}

The optimal multi-target Bayes filter propagates the multitarget posterior density $p_{k}\left(\cdot \mid Z_{1: k}\right)$ in time using recursion:

$p_{k \mid k-1}\left(X_{k} \mid Z_{1: k-1}\right)=\int f_{k \mid k-1}\left(X_{k} \mid X\right) p_{k-1}\left(X \mid Z_{1: k-1}\right) \mu_{s}(d X)$

$p_{k}\left(X_{k} \mid Z_{1: k}\right)=\frac{g_{k}\left(Z_{k} \mid X_{k}\right) p_{k \mid k-1}\left(X_{k} \mid Z_{1: k-1}\right)}{\int g_{k}\left(Z_{k} \mid X\right) p_{k \mid k-1}\left(X_{k} \mid Z_{1: k-1}\right) \mu_{s}(d X)}$

where $\mu_{s}(d X)$ is a reference measure on $\mathcal{F}(\mathcal{X})$. Computing (4)-(5) involves multiple integrals on the space $\mathcal{F}(X)$, which is intractable.

\subsection{Probability Hypothesis Filter}

To deal with intractability, the Probability Hypothesis Density (PHD) filter, instead of propagating the multi-target posterior density in time, propagates the posterior intensity, a first order statistical moment of the multi-target posterior (Mahler 2003).

For RFS $X$ on the collection of all finite subsets $\mathcal{X}$ with probability distribution $P$, the first order moment, called the intensity, is a non-negative function $v$ on $\mathcal{X}$. In essence, for each region $S \subseteq \mathcal{X}$, the integral of $v$ over that region, $\int_{S} v(\mathbf{x}) d \mathbf{x}$, gives the expected number of elements of $X$ that are in $S^{2}$. The posterior intensity approximation of the multitarget posterior, where $v_{k}$ and $v_{k \mid k-1}$ are the approximations of $p_{k}$ and $p_{k \mid k-1}$ respectively, can be propagated in time via the PHD recursion:

$v_{k \mid k-1}(\mathbf{x})=\int p_{S, k}(\zeta) f_{k \mid k-1}(\mathbf{x} \mid \zeta) v_{k-1}(\zeta) d \zeta+\gamma_{k}(\mathbf{x})$
$v_{k}(\mathbf{x})=\left[1-p_{D, k}(\mathbf{x})\right] v_{k \mid k-1}(\mathbf{x})+p_{D, k}(\mathbf{x}) v_{k \mid k-1}^{D}(\mathbf{x})$

2 The local maxima of the intensity $v$ are the local concentrations of the expected number of elements and can be used to estimate the elements of $X$. 
with

$v_{k \mid k-1}^{D}(\mathbf{x})=\sum_{\mathbf{z} \in Z_{k}} \frac{g_{k}(\mathbf{z} \mid \mathbf{x}) v_{k \mid k-1}(\mathbf{x})}{\kappa_{k}(\mathbf{z})+\int p_{D, k}(\xi) g_{k}(\mathbf{z} \mid \xi) v_{k \mid k-1}(\xi) d \xi}$

where $\gamma_{k}(\mathbf{x})$ is the intensity of the birth RFS $\Gamma_{k}$ and $\kappa_{k}(\mathbf{z})$ is the intensity of the clutter RFS $K_{k}$. The PHD filter does not involve combinatorial computations, nevertheless it does not admit closed form solutions.

\subsection{Gaussian Mixture Probability Hypothesis Filter}

The Gaussian Mixture Probability Hypothesis Density (GM-PHD) (Vo and Ma 2006) filter admits a closed form solution to the PHD recursion. Under linear, Gaussian assumptions on the target dynamics and birth processes, the posterior intensity is a Gaussian mixture of the form:

$v_{k}(\mathbf{x})=\sum_{i=1}^{J_{k}} w_{k}^{(i)} \mathcal{N}\left(\mathbf{x} ; m_{k}^{(i)}, P_{k}^{(i)}\right)$

where each Gaussian component $i$ is associated with a weight $w_{k}^{(i)}, J_{k}$ is the number of Gaussian components representing the intensity and $\mathcal{N}(\cdot ; m, P)$ denotes a Gaussian density with mean $m$ and covariance $P$.

The GM-PHD filter involves four steps: 1) prediction, where the previous intensity evolves according to the motion model and where new targets can appear 2) update, where the intensity is updated with the acquired measurements 3 ) selection, including merging and pruning, to reduce the number of Gaussian components and 4) state extraction from the posterior intensity.

\subsubsection{Prediction}

The predicted intensity at the time $k$ is a Gaussian mixture of the form:

$v_{k \mid k-1}(\mathbf{x})=v_{S, k \mid k-1}(\mathbf{x})+\gamma_{k}(\mathbf{x})$

where $v_{S, k \mid k-1}(\mathbf{x})$ is the survival intensity:

$v_{S, k \mid k-1}(\mathbf{x})=p_{S, k} \sum_{i=1}^{J_{k-1}} w_{k-1}^{(i)} \mathcal{N}\left(\mathbf{x} ; m_{k \mid k-1}^{(i)}, P_{k \mid k-1}^{(i)}\right)$

with $p_{S, k}$ being the probability of survival, and $\gamma_{k}(\mathbf{x})$ is the birth intensity with $J_{\gamma, k}$ components:

$\gamma_{k}(\mathbf{x})=\sum_{i=1}^{J_{\gamma, k}} w_{\gamma, k}^{(i)} \mathcal{N}\left(x ; m_{\gamma, k}^{(i)}, P_{\gamma, k}^{(i)}\right)$

The components of the survival intensity are computed from the previous intensity components according to a linear Gaussian motion model:

$m_{k \mid k-1}^{(i)}=F_{k-1} m_{k-1}^{(i)}$
$P_{k \mid k-1}^{(i)}=Q_{k-1}+F_{k-1} P_{k-1}^{(i)} F_{k-1}^{T}$

and $w_{k \mid k-1}^{(i)}=w_{k-1}^{(i)}$, where $F_{k-1}$ is the state transition matrix and $Q_{k-1}$ is the process noise covariance. The mean values of the birth intensity components, $m_{\gamma, k}^{(i)}$, represent places, where new targets are likely to appear.

\subsubsection{Update}

Given a set of measurements $Z_{k}$, the posterior intensity is updated as follows:

$v_{k}(\mathbf{x})=v_{T, k}(\mathbf{x})+\sum_{\mathbf{z} \in Z_{k}} v_{D, k}(\mathbf{x} ; \mathbf{z})$

where

$v_{T, k}(\mathbf{x})=\sum_{i=1}^{J_{k \mid k-1}}\left(1-p_{D, k}\right) w_{k \mid k-1}^{(i)} \mathcal{N}\left(\mathbf{x} ; m_{k \mid k-1}^{(i)}, P_{k \mid k-1}^{(i)}\right)$

$v_{D, k}(\mathbf{x}, \mathbf{z})=\sum_{i=1}^{J_{k \mid k-1}} w_{k}^{(i)}(\mathbf{z}) \mathcal{N}\left(\mathbf{x} ; m_{k \mid k}^{(i)}(\mathbf{z}), P_{k \mid k}^{(i)}\right)$

where $p_{D, k}\left(m_{k \mid k-1}^{(i)}\right)$ is the state-dependent probability of detection. Intuitively, $v_{T, k}(\mathbf{x})$ is the missed-detection term, where the weight of each Gaussian component of the predicted intensity is discounted according to $p_{D, k}$ (see Equation 15). The $v_{D, k}(\mathbf{x} ; \mathbf{z})$ term, one for each measurement $\mathbf{z} \in Z_{k}$, is the detection term, which provides closed form expressions for computing the means, covariances and weights of $v_{k}$ from those of $v_{k \mid k-1}$ when a new set of measurements arrives. The complete expressions for $w_{k}^{(i)}, m_{k \mid k}^{(i)}$ and $P_{k \mid k}^{(i)}$ in Eq. (16) are:

$w_{k}^{(i)}(\mathbf{z})=\frac{p_{D, k} w_{k \mid k-1}^{(i)} q_{k}^{(i)}(\mathbf{z})}{\kappa_{k}(\mathbf{z})+p_{D, k} \sum_{l=1}^{J_{k \mid k-1}} w_{k \mid k-1}^{(l)} q_{k}^{(l)}(\mathbf{z})}$

$m_{k \mid k}^{(i)}(\mathbf{z})=m_{k \mid k-1}^{(i)}+K_{k}^{(i)}\left(z-H_{k} m_{k \mid k-1}^{(i)}\right)$

$P_{k \mid k}^{(i)}=\left[I-K_{k}^{(i)} H_{k}\right] P_{k \mid k-1}^{(j)}$

with

$q_{k}^{(i)}(\mathbf{z})=\mathcal{N}\left(\mathbf{z} ; H_{k} m_{k \mid k-1}^{(i)}, U_{k}+H_{k} P_{k \mid k-1}^{(i)} H_{k}^{T}\right)$

$K_{k}^{(i)}=P_{k \mid k-1}^{(i)} H_{k}^{T}\left(H_{k} P_{k \mid k-1}^{(i)} H_{k}^{T}+U_{k}\right)^{-1}$

where $H_{k}$ is the observation matrix, $U_{k}$ is the observation noise covariance and $\kappa_{k}(\mathbf{z})$ is the expected clutter level. 


\subsubsection{Selection}

The update step yields a quadratic increase in the number of Gaussian components the posterior intensity is composed of. To keep the problem tractable, components with weak weights are pruned: $I=\left\{i=1, \ldots, J_{k} \mid w_{k}^{(i)}>T_{S}\right\}$.

Furthermore, all Gaussian components close to each other are merged into a single Gaussian as follows. At first, a Gaussian component with the highest weight is selected with $j=\operatorname{argmax}_{i \in I} w_{k}^{(i)}$. Then all Gaussian components within the Mahalonobis distance $U$ from $j$ are forming a set of Gaussian components:

$L=\left\{i \in I \mid\left(m_{k}^{(i)}-m_{k}^{(j)}\right)^{T}\left(P_{k}^{(i)}\right)^{-1}\left(m_{k}^{(i)}-m_{k}^{(j)}\right) \leq U_{S}\right\}$

that are merged into a single component:

$\tilde{w}_{k}^{(l)}=\sum_{i \in L} w_{k}^{(i)}, \quad \tilde{m}_{k}^{(l)}=\frac{1}{\tilde{w}_{k}^{(l)}} \sum_{i \in L} w_{k}^{(i)} m_{k}^{(i)}$,

$\tilde{P}_{k}^{(l)}=\frac{1}{\tilde{w}_{k}^{(l)}} \sum_{i \in L} w_{k}^{(i)}\left(P_{k}^{(i)}+\left(\tilde{m}_{k}^{(l)}-m_{k}^{(i)}\right)\left(\tilde{m}_{k}^{(l)}-m_{k}^{(i)}\right)^{T}\right)$

Finally, the number of Gaussian components is truncated to $J_{\max }$ components with the highest weights, resulting in a posterior intensity shaped as a Gaussian mixture:

$v_{k}(\mathbf{x})=\sum_{i=1}^{J_{k}} w_{k}^{(i)} \mathcal{N}\left(x ; m_{k}^{(i)}, P_{k}^{(i)}\right)$

\subsubsection{State extraction}

The means of the Gaussian components are the local maxima of the posterior intensity $v_{k}$. Extraction of multi-target state estimates comes down to selection of the Gaussian means that have weights greater than a threshold $T_{S E}$.

\section{Problem Statement}

We address the problem of multi-robot tracking to provide absolute position estimates necessary for a team of robots to control and keep a desired formation geometry. The multirobot system consists of $\Delta$ robots $R_{1}, \ldots, R_{\Delta}$. The formation includes one leader robot, which moves on a pre-defined trajectory, and $\Delta-1$ follower robots, all of which know the desired formation geometry, i.e. the desired range and bearing they should keep with respect to their neighbors, including the leader.

Each robot $R_{i}$ independently estimates its own position $\mathbf{p}_{i}=\left[x_{i}, y_{i}\right]$ and orientation $\alpha_{i}$ in a two-dimensional, GNSSdenied environment, based on a known map and onboard sensing. Since all the robots share the same map, they all share a common global coordinate frame $I_{W}$. Each robot $R_{i}$ is equipped with sensors that provide range and bearing to the other robots in its local reference frame $I_{R_{i}}$, but the measurements do not include IDs of the detected robots. Since the relation of $I_{R_{i}}$ w.r.t. $I_{W}$ is known to $R_{i}$, the range and bearing sensor measurement $j$ can be expressed in the global frame as $\mathbf{z}_{j}$ to constitute the measurements set $Z_{k}$. Because of the lack of IDs, the followers do neither have the means to distinguish among the neighbors, nor can they tell apart a leader from a follower based on the measurements alone. Therefore, the formation orientation is defined in $I_{W}$, which is the only frame known to all the robots. The frame of the leader, $I_{L}$, cannot be used for that purpose, as it is not known to the robots at all times. Robots are capable of communicating with each other their global self-localization positions in $I_{W}$, but the communication is not reliable enough to be used as the only means to maintain the formation.

The state $\mathbf{x}_{j}=\left[x_{j}, y_{j}, \dot{x}_{j}, \dot{y}_{j}\right]$ of each target robot in the global reference frame $I_{W}$ consists of its position and velocity. Each target follows the linear Gaussian dynamical model with:

$F=\left[\begin{array}{cc}I_{2} & \delta I_{2} \\ 0_{2} & I_{2}\end{array}\right], \quad Q=\sigma_{f}^{2}\left[\begin{array}{ll}\frac{\delta^{4}}{4} I_{2} & \frac{\delta^{3}}{2} I_{2} \\ \frac{\delta^{3}}{2} I_{2} & \delta^{2} I_{2}\end{array}\right]$

where $I_{n}$ and $0_{n}$ denote, respectively, the $n \times n$ identity and zero matrices, $\delta$ is the time step, and $\sigma_{f}^{2}$ is the standard deviation of the process noise. The sensor measurement $\mathbf{z}_{j}=\left[z_{j}^{x}, z_{j}^{y}\right]^{T}$, expressed in the global frame $I_{W}$ is a noisy version of the position of a target robot $R_{j}$ and follows a linear Gaussian observation model with $H=\left[I_{2}, O_{2}\right]$, $U=\sigma_{\varepsilon}^{2} I_{2}$, where $\sigma_{\varepsilon}^{2}$ is the standard deviation of the measurement noise. The communicated information sent by $R_{j}$ to the receiving robot $R_{i}$ includes a position $\mathbf{p}_{j}$ in $I_{W}$. Multitarget tracking is performed in the global frame $I_{W}$.

\section{GM-PHD Filter with Formation Information}

In this work we introduce the FI-GM-PHD filter. The FIGM-PHD filter consists of two steps for supplementing additional Gaussian components to the filter intensity:

1. The inception step - incorporation of communication data;

2. The coalition step - using spatial configuration of the formation as a prior for the PHD filter.

To provide the robots with the necessary information to maintain the formation, each follower robot $R_{i}$ runs its own FI-GM-PHD filter. The leader robot moves independently of the other robots and therefore does not rely on other robot estimates.

The filters are decentralized from the computational viewpoint, as they are run locally and independently by 
the different follower robots. Role assignment and formation control procedures are also run individually by the follower robots. The filters are centralized from the information viewpoint, because each follower runs the FI-GM-PHD filter with information about the locations of all its teammates.

\subsection{Prediction in the FI-GM-PHD Filter}

The components of the survival intensity $v_{S, k \mid k-1}(\mathbf{x})$ constituting the predicted intensity $v_{k \mid k-1}(\mathbf{x})$ (Eq. 9) at the time $k$ are computed from the previous intensity components according to a linear Gaussian motion model: $m_{k \mid k-1}^{(i)}=F_{k-1} m_{k-1}^{(i)}$ and $P_{k \mid k-1}^{(i)}=Q_{k-1}+F_{k-1} P_{k-1}^{(i)} F_{k-1}^{T}$ (Eq. 12 and Eq. 13 respectively). The motion model of a target with the state $\mathbf{x}_{j}=\left[x_{j}, y_{j}, \dot{x}_{j}, \dot{y}_{j}\right]$ composed of position and velocity is a linear Gaussian dynamical model with the state transition matrix $F=\left[I_{2}, \delta I_{2} ; 0_{2}, I_{2}\right]$ and the process noise covariance $Q=\left[\frac{\delta^{4}}{4} I_{2}, \frac{\delta^{3}}{2} I_{2} ; \frac{\delta^{3}}{2} I_{2}, \delta^{2} I_{2}\right]$, described in detail in Section 4. At initialization, the filter run by robot $R_{i}$ is supplied with a birth RFS $\gamma_{0}(\mathbf{x})$ at the initial detections. At time $k \neq 0$, birth intensity in Eq. 9 is $\gamma_{k}(\mathbf{x})=\emptyset$.

\subsection{Update in the FI-GM-PHD Filter}

The update step in the FI-GM-PHD filter differs from the procedure of the original filter described in Sec. 3.3.2 with regard to the source of the measurements. While in the GM-PHD filter the set of measurements $Z_{k}$ consists of direct measurements obtained from the sensors of the tracking robot, the detection term $v_{D, k}$ in the FI-GM-PHD filter also includes communicated state information, added in what we refer to as the inception step.

\subsubsection{Measurement Update}

The missed-detection term $v_{T, k}(\mathbf{x})$ constituting the update intensity in Eq. 14 is calculated using the predicted intensity $v_{k \mid k-1}(\mathbf{x})$, discounted according to the probability of detection $p_{D, k}$ as shown in Eq. 15. The state-dependent probability of detection used in this work is described in detail in Sec. 5.2.3; it suggest how likely it is that the tracked robot is detected given the occlusions and its position with respect to the field of view of the tracking robot. The detection term $v_{D, k}(\mathbf{x} ; \mathbf{z})$ (Eq. 16), one for each measurement of the position $\mathbf{z}_{j}=\left[z_{j}^{x}, z_{j}^{y}\right]^{T}$ of the target $R_{j}$, follows a linear Gaussian observation model with the observation matrix $H=\left[I_{2}, 0_{2}\right]$ and the observation noise covariance $U=\sigma_{\varepsilon}^{2} I_{2}$. The sensor measurements in $Z_{k}$ are obtained from onboard range and bearing sensors described in Sec 7.2, where the range and bearing data is used to determine the position of target $R_{j}$ in the global frame $I_{W}$ as explained in Sec. 4.

\subsubsection{Inception of the Communicated Data}

Even when communication between the robots is possible, it may suffer of message losses, be of low rate or break occasionally. For the receiving robot $R_{i}$ to perform inception in the update step at time $k$, the communicated position information $\mathbf{p}_{j, k}$, one per each communicating neighbor robot $j=1, \ldots, \Delta_{k}$, forms a measurement $\mathbf{z}_{k}^{(j)}$. The measurement is added to $Z_{k}$ to form a new measurement set:

$Z_{k}:=Z_{k} \cup \sum_{j=1}^{\Delta_{k}} \mathbf{z}_{k}^{(j)}$

There is no need to associate the position messages with the existing Gaussian components from the prediction step, as the PHD filter does not require data association, but each measurement generates a new set of components updated from the predicted intensity (see Eq. 16). In the general case including communicated measurements is not suitable for the update step, because if only a fraction of the robots is exchanging the information, then performing the update would delete from the output the tracks of the robots that did not communicate. This can be prevented by ensuring that when merging the Gaussian components generated by the transmitted data of the robots that $d o$ communicate and the Gaussian components from the prediction step that might correspond to those robots, the weight of the merged component always remaining between $(0,1)$.

By curbing the merged weight, the predicted components that are not strengthened by additional communicated data are prevented from pruning. The selection step thus includes an additional merging procedure. Now, after performing the standard merging step (Eq. 22 to Eq. 23), we again select a Gaussian component with the highest weight $j=\operatorname{argmax}_{i \in I} w_{k}^{(i)}$. Then all the components within an Euclidean distance $U$ between the position part of the Gaussian mean, $m_{0: 1}$, and the position part of the mean of $j$ form the set $L^{\prime}=\left\{i \in I \mid\left\|m_{0: 1, k}^{(i)}-m_{0: 1, k}^{(j)}\right\| \leq 2 r_{r}\right\}$, where $2 r_{r}$ is the robot diameter. The set $L^{\prime}$ is merged into a single component with the weight $\tilde{w}_{k}^{\prime(l)}=\min \left(\sum_{i \in L^{\prime}} w_{k}^{(i)}, 1\right)$. The calculation of $\tilde{m}_{k}^{\prime(l)}$ and $\tilde{P}_{k}^{\prime(l)}$ proceeds as in Eq. 23.

Note that if the model for the communicated measurements differed from the model of the sensor-based measurements, Eqs. 17-19 would have to be applied with each measurement updated according to its respective model.

\subsubsection{Probability of Detection}

In order to reduce the risk of losing track of a robot when it enters an occluded area or escapes the field of view of the detecting robot, the probability of detection $p_{D, k}\left(m_{k \mid k-1}^{(i)}\right)$ is state dependent. Constant probability of detection would 


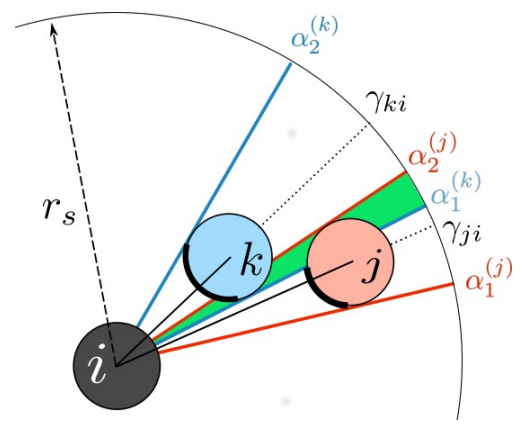

Fig. 2: Illustration of the occlusions and field of view model. The detecting robot $R_{i}$ determines the degree of occlusion of the robot $R_{j}$. Angles $\left[\alpha_{1}^{(o)}, \alpha_{2}^{(o)}\right]$ are the boundaries of the region occluded by object $o$. The green region is the intersection of the occlusion regions of $R_{j}$ and $R_{k}$.

result in quick decrease of the weights of the components without associated measurements.

The method for determining $p_{D, k}\left(m_{k \mid k-1}^{(i)}\right)$ is summarized in Algorithm 1 and illustrated in Figure 2. If the estimated position $\hat{\mathbf{p}}_{j}$ of the tracked robot $R_{j}$ is outside the sensing range $r_{s}$ of the detecting robot $R_{i}$, a minimum detection probability $p_{D}^{\text {min }}$ is assigned (line 4 in Algorithm 1). Additionally, the object of interest is considered occluded if any other object $k$ is located between the detecting robot and the object of interest. In this case, the decrease in the probability of detection is proportional to the weight of the occluding object and proportional to the estimated degree of occlusion.

In the Inception step (Sec. 5.2.2), the probability of detection associated with the components stemming from the communicated measurements $p_{D}^{I n c}$ is state-independent and constant.

\subsection{The Projected Formation State}

Given the absolute position $\mathbf{p}_{i, k}=\left[x_{i, k}, y_{i, k}\right]$ of the robot $R_{i}$ at time $k$, the position of the robot $R_{j}$ is projected in the global frame $I_{W}$ based on the desired formation geometry:

$\left[\begin{array}{l}h_{i j, k}^{x} \\ h_{i j, k}^{y}\end{array}\right]=\left[\begin{array}{l}b_{i j}^{x} \\ b_{i j}^{y}\end{array}\right]+\left[\begin{array}{l}x_{i, k} \\ y_{i, k}\end{array}\right]$

where $b_{i j}$ is a bias, i.e. known desired spacing between the robot $R_{i}$ and $R_{j}$. The collection of the projected positions with respect to the robot $R_{i}$ of all the other robots in the formation is denoted by:

$h_{i, k}:=\left\{\left\{h_{i j, k}^{x}, h_{i j, k}^{y}\right\}, \mid j=1, \ldots, \Delta ; j \neq i\right\}$

where $\Delta$ is the number of robots in the formation and so, $\left|h_{i, k}\right|=\Delta-1$.

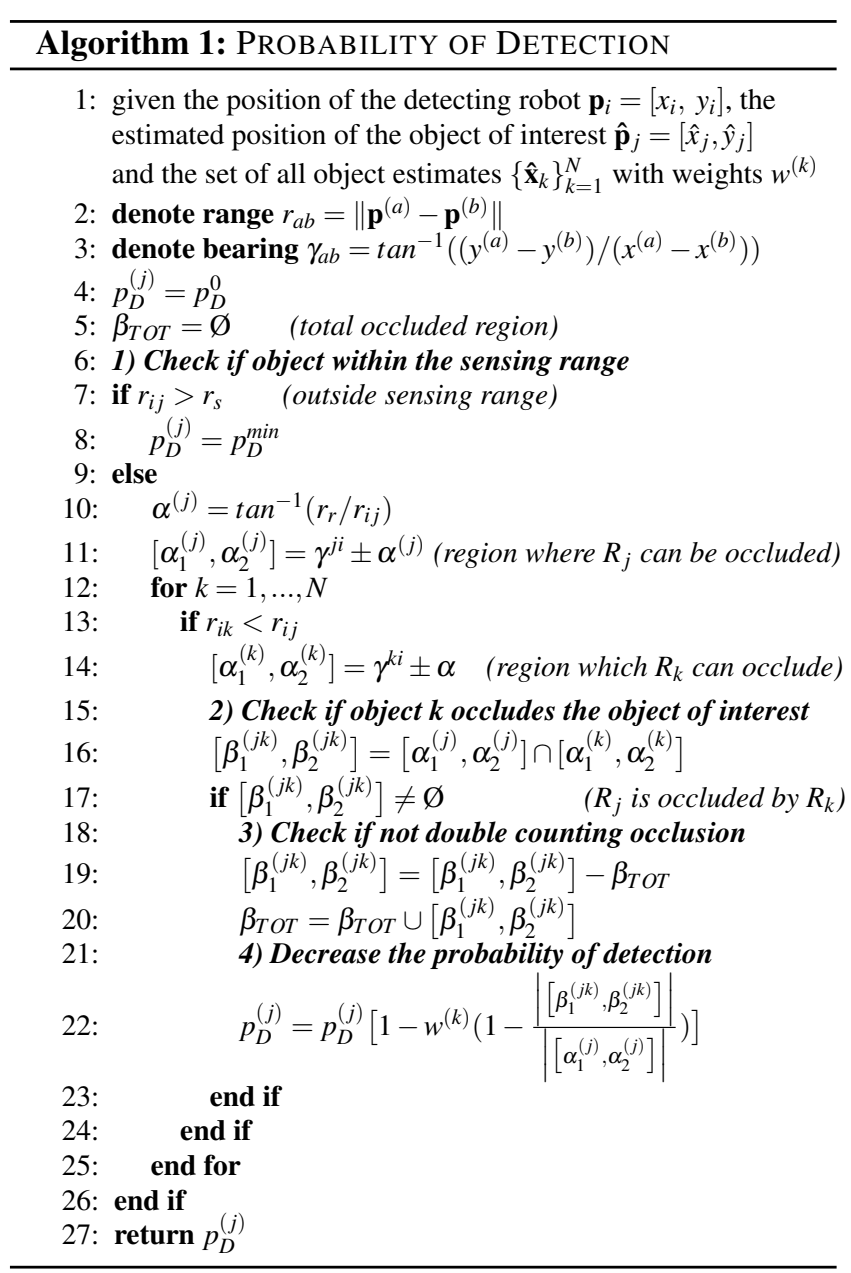

\subsection{Coalition of the Projected Formation States}

The coalition step extends the GM-PHD filter with an additional block, added after the update step. It combines the intensities obtained during the update step with the coalition intensity derived from the projected formation states. Thus, the Gaussian components constituting the coalition intensity serve as an outline of where the tracked robots are to be expected.

The coalition step is detailed in Algorithm 2. The set of states $h_{i, k}$ projected by the robot $R_{i}$ is used to approximate the means of the components of the coalition intensity $v_{\zeta, k}$ as follows. For each tracked robot $R_{j}$ the mean is placed at the projected position of that robot $m_{\zeta, k}^{(j)}=\left[h_{i j, k}^{x}, h_{i j, k}^{y}, 0,0\right]^{T}$, where $j=1, \ldots, \Delta ; j \neq i$ and so $J_{\zeta, k} \stackrel{\zeta, k}{=} \Delta-1$. Each component $j=1, \ldots, J_{\zeta, k}$ of the coalition intensity $v_{\zeta, k}$ at time $k$ is given an initial budget $\Phi_{\zeta, k}^{(j)}=\Phi_{\zeta, 0}$. Then, all the components forming the posterior intensity $v_{k}(\mathbf{x}), l=1, \ldots, J_{k}$, are compared against $j$ to find the matching that maximizes some criteria (line 7 of Algorithm 2). We choose to put emphasis on minimizing the distance between the position part of the component means $\left(m_{0: 1}\right.$ corresponds to the position part of 


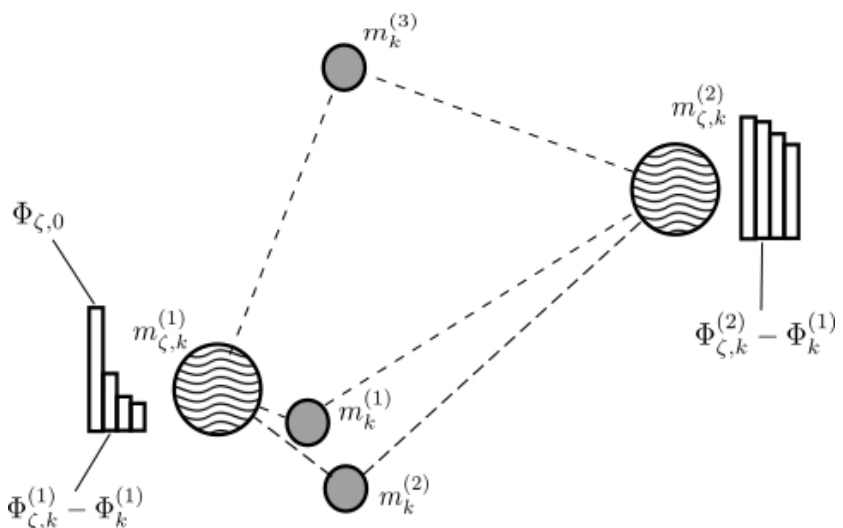

Fig. 3: Illustration of the coalition step. Three posterior intensity components, $m_{k}^{(1)}, m_{k}^{(2)}, m_{k}^{(3)}$ are compared against two coalition components, $m_{\zeta, k}^{(1)}, m_{\zeta, k}^{(2)}$ with the corresponding budgets $\Phi_{\zeta, k}^{(1)}, \Phi_{\zeta, k}^{(2)}$. Closeness of the $m_{\zeta, k}^{(1)}$ to the $m_{k}^{(1)}$ decreases significantly its budget, while for $m_{\zeta, k}^{(2)}$, the budget does not become depleted and a novelty is created.

the state, while $m_{2: 3}$ corresponds to the velocity part of the state), while choosing components of the posterior intensity with significant weights. By sorting the posterior intensity components according to the $o_{k}^{(j, l)}$ measure, we first evaluate the best candidates for good matching.

The components are coalesced as follows. Lines 16- 18 calculate a temporary budget for the posterior component $\left(\Phi_{k}^{(l)}\right)$ using a sigmoid function $f \in\langle 0,1\rangle$ of the distance between the means of the components (line 15). If the distance is small, the possibility that both components correspond to the same target is high and the components $j$ and $l$ are coalesced to form a new Gaussian component, with a mean of intensity being a combination of the means of the two, the covariance and the weight being the covariance and the weight of $l$ modified as a function of the divergence. If the distance between $j$ and $l$ is large, the likeliness that $l$ is associated with $j$ is small and $l$ is propagated further with little change. This diverse behavior is assured by using a sigmoid-shaped function of the distance when comparing the components.

The budget of the coalition component is decreased with every posterior component that has been found close to it, and has two major advantages. Firstly, it limits the number of new components that can originate around it. Secondly, a budget left at the end of iteration indicates that one of the coalition components did not have a corresponding component in the posterior, whether because of the missed detection, occlusion, or field of view limits. In that case, a new component, called the novelty is created with the mean at the area where a robot is expected to be. Only the coalition components are allocated the initial budget; the components

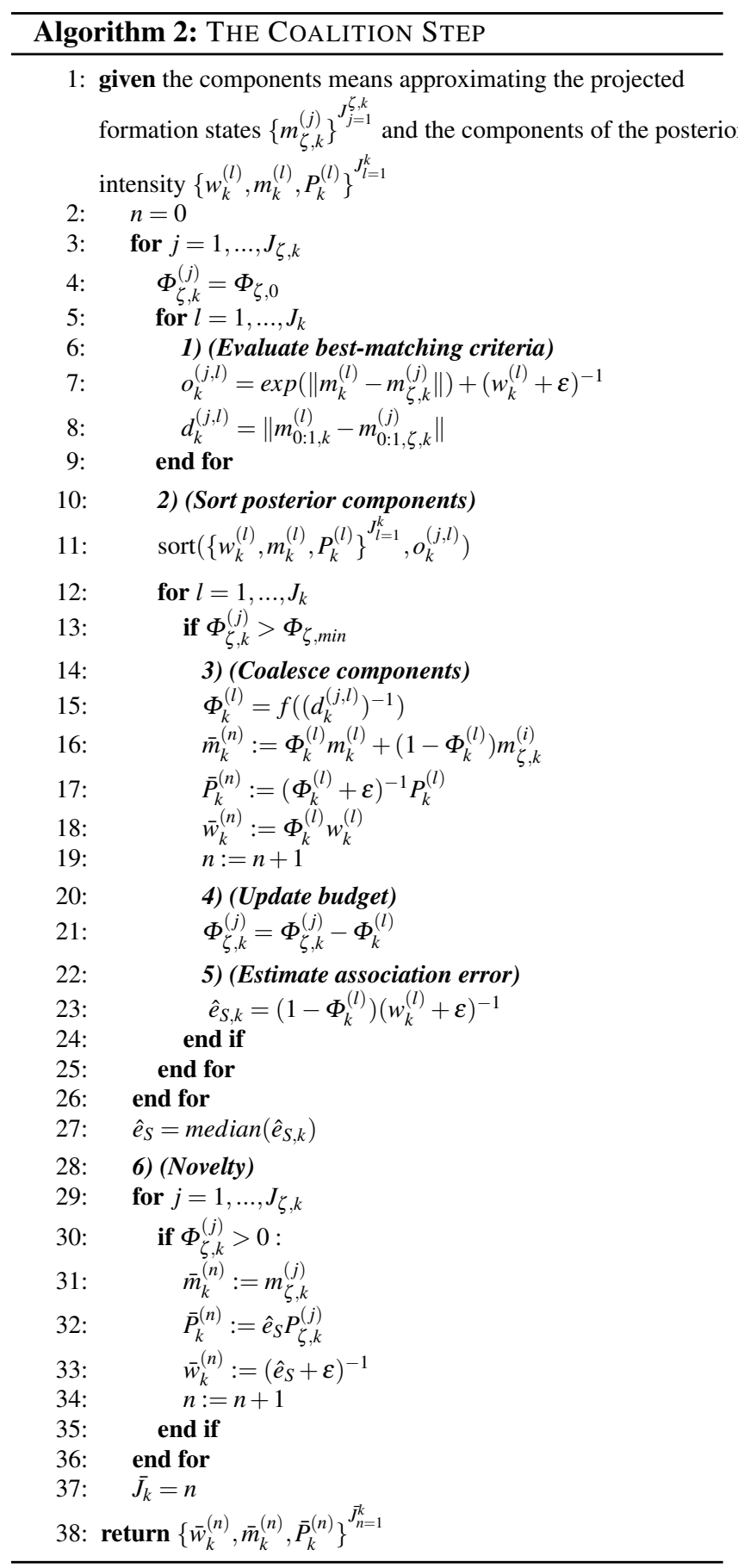

of the posterior intensity come with an associated weight and their budget is computed during the coalition process (line 15).

While in our previous work Wasik et al (2017a) we assumed that the formation always stays close-to-desired, here we relax this assumption by moderating the importance of the novelty components according to how well the matching between the posterior and the coalition components has been performed on the whole. The matching is estimated us- 
ing the association error $\hat{e}_{S}$, calculated in line 23 of Alg. 2, where a good matching means that a posterior component with a substantial weight is found close to a coalition component. The quality of the overall matching is used to determine the weight of the novelty components in line 33 . The larger the error, the lower the weight of the novelty component. In case the overall matching is poor, the weights of the coalition components are negligible and become discarded in the prediction step of the next iteration. This property modulates the impact of the coalition step on the role assignment procedure, making the latter rely on a) the Gaussian components of the full FI-GM-PHD filter when the matching is good or b) the Gaussian components created based on communicated and sensed data otherwise, for example when formation shape is not perfect.

As an example, depicted in Figure 3, consider three posterior intensity components, $m_{k}^{(1)}, m_{k}^{(2)}, m_{k}^{(3)}$ compared against two coalition components, $m_{\zeta, k}^{(1)}, m_{\zeta, k}^{(2)}$ with the corresponding budgets $\Phi_{\zeta, k}^{(1)}, \Phi_{\zeta, k}^{(2)}$ initially set to $\Phi_{\zeta, 0}$. Closeness of $m_{k}^{(1)}$ to $m_{\zeta, k}^{(1)}$ decreases significantly its budget $\Phi_{\zeta, k}^{(1)}$, and a new component is created as a combination of the two. For $m_{\zeta, k}^{(2)}$, the budget does not become depleted and a novelty is created. Finally, component $m_{k}^{(3)}$ has no close correspondence in $v_{\zeta_{k}}$ and it is propagated with no modification.

The coalition step and the inception step supplement the intensity with additional Gaussian components. In case the robot has not been tracked, adding a new Gaussian component creates a new target based on the high probability that the target is there. This is analogous to the birth process of the GM-PHD filter in Vo and Ma (2006). In case the robot has already been tracked, adding a new Gaussian component that corresponds to that robot increases the likelihood of the robot being present at that position, provided that the state extraction takes into account the fact that multiple targets cannot occupy the same physical position, i.e. the components with weight above one are extracted as single targets.

\section{Tracking for Formation Control}

\subsection{Graph-Based Formation Control}

Details of the formation control algorithm can be found in Wasik et al (2016a). The formation is comprised of $\Delta$ holonomic robots including one leader robot that moves on a predefined trajectory. In contrast with our previous work on FI-GM-PHD filter (Wasik et al 2017a), the position of the leader is not known globally to all the robots, but the robots must track the leader in order to follow it in the formation.

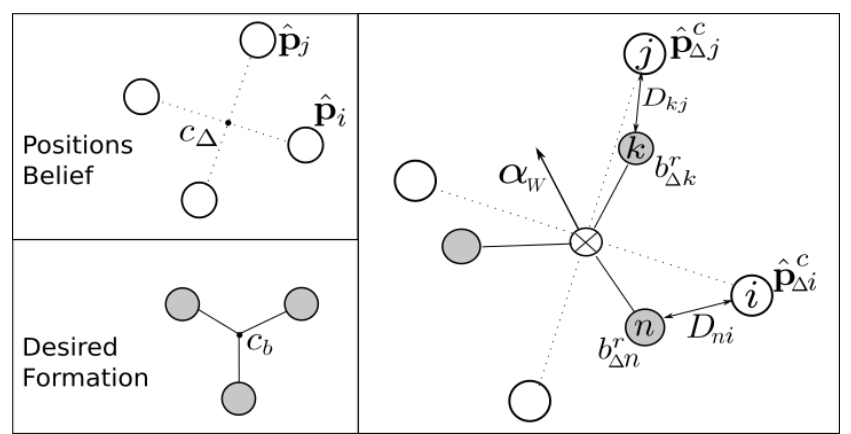

Fig. 4: Illustration of the role assignment step. Center of the estimates $\mathbf{c}_{\Delta}$ and center of the formation $\mathbf{c}_{b}$ are brought to a common reference frame at the origin, where the translated position estimates are $\hat{\mathbf{p}}_{\Delta}^{c}$, and the bias, translated and rotated according to the formation orientation $\alpha_{W}$, is $\mathbf{b}_{\Delta}^{r} . D_{n i}$ is the cost of associating bias $\mathbf{b}_{n}$ with estimate $\hat{\mathbf{x}}_{i}$.

For a robot $R_{i}$, the formation control is achieved as:

$$
\begin{aligned}
& \dot{x}_{i}=\frac{1}{\left|\sum_{j} \mathcal{L}_{i j}\right|} \sum_{i \sim j}\left[-\mathcal{L}_{i j}\left(r_{i j}(t) \cos \left(\gamma_{i j}(t)\right)-b_{i j}^{x}(t)\right)\right] \\
& \dot{y}_{i}=\frac{1}{\left|\sum_{j} \mathcal{L}_{i j}\right|} \sum_{i \sim j}\left[-\mathcal{L}_{i j}\left(r_{i j}(t) \sin \left(\gamma_{i j}(t)\right)-b_{i j}^{y}(t)\right)\right]
\end{aligned}
$$

where $\mathcal{L}$ is a non-stationary Laplacian, $r_{i j}$ and $\gamma_{i j}$ are the Euclidean relative range and the bearing between the robots $R_{i}$ and $R_{j}$ respectively. The bias vectors $\mathbf{b}_{i}^{x}, \mathbf{b}_{i}^{y} \in \mathbb{R}_{i}^{\Delta_{i}^{L}}$ define the desired robot-leader and inter-robot distances along the $x$ and $y$ axes respectively. Finally, the robots are omnidirectional, so the followers heading is decoupled from the velocity control. Since the pose of the leader is not globally shared, the followers align their orientation to the positive y-axis of the map frame $I_{W}{ }^{3}$.

\subsection{Role Assignment}

The role assignment procedure finds a permutation that assigns the estimates and the position of the detecting robot to the target positions in the formation. Although we use the graph-based framework notation, the method can be generalized to other formation algorithms. The procedure is sketched in Algorithm 3. Consider a formation with its geometry uniquely determined by a bias matrix $\mathbf{b}_{\Delta}$, a set of desired inter-robot distances. The heading of the formation is defined in a global reference frame and is assumed to be known by all the robots. In our case the formation orientation $\alpha_{W}$ is along the y-axis of the world frame. The bias and the estimates are brought to a common reference frame by matching the center of the rotated global bias $\mathbf{b}_{\Delta}^{r}$ with

3 The robots self-localize on a known map, therefore the orientation of the body with respect to the map frame is estimated online. 


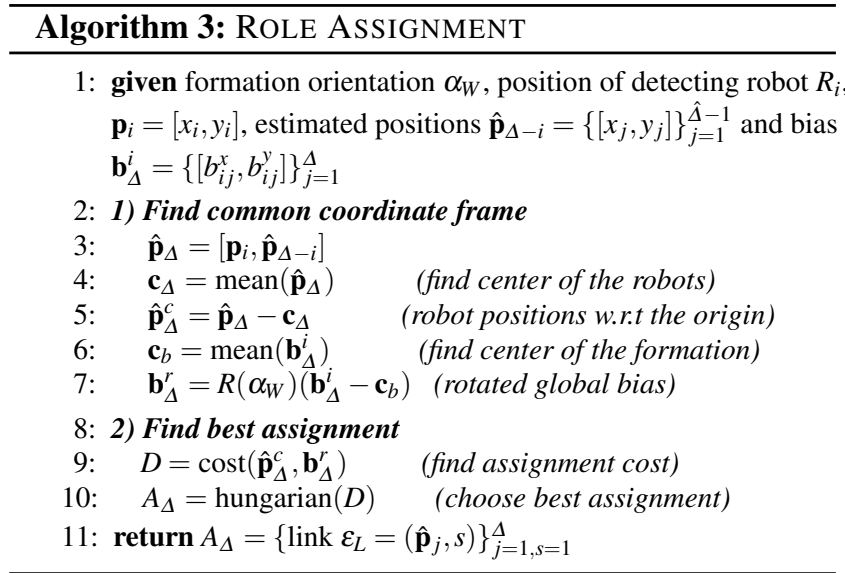

the center of the estimated positions $\hat{\mathbf{p}}_{\Delta}^{c}$ (see Fig. 4). The combination of the estimates and the roles resulting in the smallest cost is computed using the Hungarian algorithm (Kuhn 1955). The role assignment procedure, run by the robot $R_{i}$, provides a set of links, $A_{\Delta}=\left\{\varepsilon_{L}=\left(\hat{\mathbf{p}}_{i}, s\right),\right\}_{s=1}^{\Delta}$. The link $\varepsilon_{L}=\left(\hat{\mathbf{p}}_{j}, s\right)$ is used for correct matching of the bias $b_{i s}(t)$ with $r_{i j}(t)$ and $\gamma_{i j}(t)$ in Eq. 29, while its cost gives the confidence about the assignment. More precisely, estimate $\hat{\mathbf{x}}_{j}$ (akin to position $\hat{\mathbf{p}}_{j}$ ) is associated with role " $s$ " and its subscript is changed to $\hat{\mathbf{x}}_{s}$, with the range $r_{i s}$ and the bearing $\gamma_{i s}$ with respect to the robot $R_{i}$. The estimate $\hat{\mathbf{x}}_{s}$ is coupled with bias $b_{i s}$ that corresponds to the " $s$ th" place in the formation.

The role assignment procedure provides the tracking robot $R_{i}$ with its own role. In the next iteration of the FIGM-PHD filter this role is used to determine the projected formation state that affects the coalition step, which in turn after state extraction is used for the next role assignment. Since the coalition step introduces novelty components for robots that can neither be communicated with nor tracked, it is important to note that without the fundamental assumption of our work, namely that only a subset of robots experiences communications outage at the same time, errors are likely to occur and have a tendency to escalate. With relatively small teams of robots, the formation recovers from sporadic inconsistencies.

\section{Experiments}

Experiments are performed in the high-fidelity robotic simulator Webots (Michel 2004), with holonomic MBot robots of diameter $0.65 \mathrm{~m}$ shown in Fig. 5. The robots are equipped with two LIDARs providing $360^{\circ}$ field of view and sensing range $4 \mathrm{~m}$, which are accurately simulated and calibrated using real data. Robots self-localize using the $\mathrm{AMCL}^{4}$ package from ROS, with the accuracy of approximately 10 to

4 AMCL (http://wiki.ros.org/amcl)

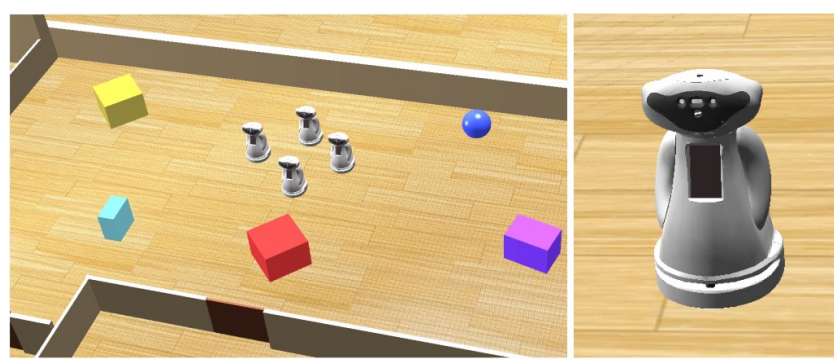

Fig. 5: A screenshot from the Webots simulator. The MBot robot (right) and the simulated indoor environment (left).

$20 \mathrm{~cm}$ of position, and around $5 \mathrm{deg}$ of orientation. Path planning performed by the leader robot is achieved using the Fast Marching Method (FMM) (Ventura and Ahmad 2015). Each robot uses a Dynamic Window Approach (DWA) for low-level reactive collision avoidance. The method finds a velocity candidate that is the closest to the desired output velocity, in this case closest to the formation control output in Eq. 29, but also guarantees collision-free trajectory. See Wasik et al (2016a) for details.

\subsection{Implementation}

The tracking filter is run in a global frame $I_{W}$. The tracking parameters of the filter are as follows. State of the target is $\mathbf{x}_{j}=\left[x_{j}, y_{j}, \dot{x}_{j}, \dot{y}_{j}\right]$. Each target has the survival probability $p_{S, k}=0.95$, and follows the linear Gaussian dynamical model (see Sec. 4) with $\sigma_{f}^{2}=1.0$, which accounts for motion uncertainty associated with holonomic motion of robots, and $\delta=0.5 s$. The measurement $\mathbf{z}_{i}=\left[z_{i}^{x}, z_{i}^{y}\right]^{T}$ is a noisy version of the position and follows a linear Gaussian observation model with the standard deviation of the position $\sigma_{\varepsilon}^{2}=0.2$, determined empirically in simulation.

At initialization, the filter run by robot $R_{i}$ is supplied with a birth RFS at the initial detections with the covariance $P_{\gamma, 0}=\operatorname{diag}\left(\left[\sigma_{\varepsilon}^{2}, \sigma_{\varepsilon}^{2}, \sigma_{\gamma}^{2}, \sigma_{\gamma}^{2}\right]^{T}\right)$, where $\sigma_{\gamma}^{2}=1.0$, and the weight $w_{\gamma, 0}=1.0$. We choose $\sigma_{\varepsilon}^{2}$ as it is the expected measurement error and $\sigma_{\gamma}^{2}$ as the measurements do not include the velocity. The parameters for merging and pruning are: $J_{\max }=10, U_{S}=0.3, T_{S}=10^{-4}$ and we select Gaussian components above $T_{S E}=0.5$. Furthermore, to take into account physical dimensions of the robots, the selection step (see Eq. 22) additionally merges components if their means are closer than twice the radius of the robot, $2 r_{r}=0.65 \mathrm{~m}$, $L=\left\{i \in I \mid\left\|m_{k}^{(i)}-m_{k}^{(j)}\right\| \leq 2 r_{r}\right\}$. Poisson distributed clutter level is $\kappa_{k}(z)=0.015$ within the sensing range of the robot. Parameters for finding detection probability are $p_{D}^{0}=0.9$, $p_{D}^{\min }=0.02$. The sensing radius $r_{s}=4.0 \mathrm{~m}$ is range of the laser scan used in the simulation. 
Inception Given that the sending robot $R_{i}$ at time $k$ communicates its estimated position $\mathbf{p}_{i, k}=\left[x_{i, k}, y_{i, k}\right]$ and the orientation, this information is used in the inception step in the form of a measurement, where $\mathbf{z}_{k}^{(i)}=\left[x_{i, k}, y_{i, k}\right]$. Robot orientation is not included in the state estimation as our current laser detection cannot provide orientation information. Constant probability of detection used in the model is $p_{D}^{I n c}=0.9$.

Coalition The coalition mean intensity is based on the projected formation state $m_{\zeta, k}^{(j)}=\left[h_{i j, k}^{x}, h_{i j, k}^{y}, 0,0\right]^{T}, j=1, \ldots, J_{\zeta, k}$. The initial budget is $\Phi_{\zeta, 0}=0.5$ and the minimal budget is $\Phi_{\zeta, \min }=0.1$. The function used for coalescing the components is $f(x)=\frac{1}{1+\exp (-10(x-0.5))}$.

\subsection{Measurement Model}

For robot detection we use an on-board relative localization system originally presented in Wasik et al (2016b). At each time step, the laser range finder point cloud points are clustered using a DBSCAN algorithm ${ }^{5}$ (Ester et al 1996). For the robot with a circular model of the base with a diameter of approximately $0.65 \mathrm{~m}$, the clusters are subject to circle fitting using a least squares solver, where the center of the circle corresponds to the center of the base of the detected robot. The coordinates are compared against a known occupancy grid map to filter out false positives stemming from known obstacles. The measurement is a position returned in the form $\mathbf{z}_{i}=\left[z_{i}^{x}, z_{i}^{y}\right]^{T}$.

\subsection{Performance Evaluation}

We study the tracking performance using the Optimal SubPattern Assignment (OSPA) metric (Schuhmacher et al 2008). OSPA is comprised of two components, one accounting for localization accuracy and the other for the cardinality error:

$\bar{o}_{p}^{(c)}(X, Y)=\left(\frac{1}{\delta}\left[\min _{\pi \in \Pi} \sum_{(i, j) \in \pi} \min \left(\left\|x_{i}, y_{j}\right\|, c\right)^{p}+c^{p}(|n-m|)\right]\right)^{1 / p}$

where $n=|X|, m=|Y|$ and $\delta=\max (m, n)$. $\Pi$ is the best permutation between $X$ and $Y$ found using Hungarian algorithm, $p$ penalizes the estimated position error and $c$ is the cut-off parameter for penalizing cardinality errors. In our experiments, OSPA is computed between the ground truth positions and the estimated positions, unless stated otherwise. Each robot calculates the OSPA metric individually based on the outcome of its own instance of the filter. We present the mean results of the robots, averaged over the runs.

\footnotetext{
5 Wasik et al (2016b) uses sliding window, nearest neighbor classification for that purpose.
}

The formation error is the average difference between the desired distances and the actual distances between the robots in the formation:

$e_{F}=\left(\left|\sum_{j} \mathcal{L}_{i j}\right|\right)^{-1} \sum_{i \sim j}\left|\left(\mathbf{x}_{i}-\mathbf{x}_{j}\right)-b_{i j}\right|$

\section{Scenarios}

With four scenarios, we conduct a thorough evaluation of the FI-GM-PHD filter, and present a comparison with respect to the full-communication-no-tracking situation and with respect to the standard GM-PHD filter. We study the performance of the methods under the following four scenarios: I) for tracking purposes only, i.e. the robots do not use the tracking data for control; II) for formation initialization and convergence; III) in challenging situations, where the robots navigate around obstacles scattered in the environment or the measurement error is large; IV) in realistic scenario where communications suffers from the periods of outage. For videos of the experiments, please refer to Section 11.

\subsection{Scenario I: Multi-Robot Tracking}

The dataset is collected when five robots, one leader and four followers, move in a cross-shaped formation on a circular trajectory. Robots maintain the formation using communicated self-localization information, while simultaneously collecting sensory data. With the collected data we perform multi-robot tracking with i) the standard GM-PHD filter, and with the variations of the FI-GM-PHD method: ii) with inception step only, with iii) the coalition step only, and with iv) the full FI-GM-PHD system (both inception and coalition steps). We test the methods that combine communications (ii and iv) with message drop probabilities $p_{m d}=0.0$, $p_{m d}=0.5$ and $p_{m d}=0.9$ and in a situation when two robots are not communicating and the other robots communicate with $p_{m d}=0.0, p_{m d}=0.5$ and $p_{m d}=0.9$. For each experiment we perform 10 sequential runs, each lasting approximately $180 \mathrm{~s}$.

\subsection{Scenario II: Initialization}

We generate ten worlds (corresponding to ten experimental runs) with four robots placed at random initial positions and with random orientations. The sensing network is initially connected. In the experiments, both the tracking and the control steps of our method are applied, which means that the tracking data is used for initialization (bringing the robots from random initial positions to positions in the 


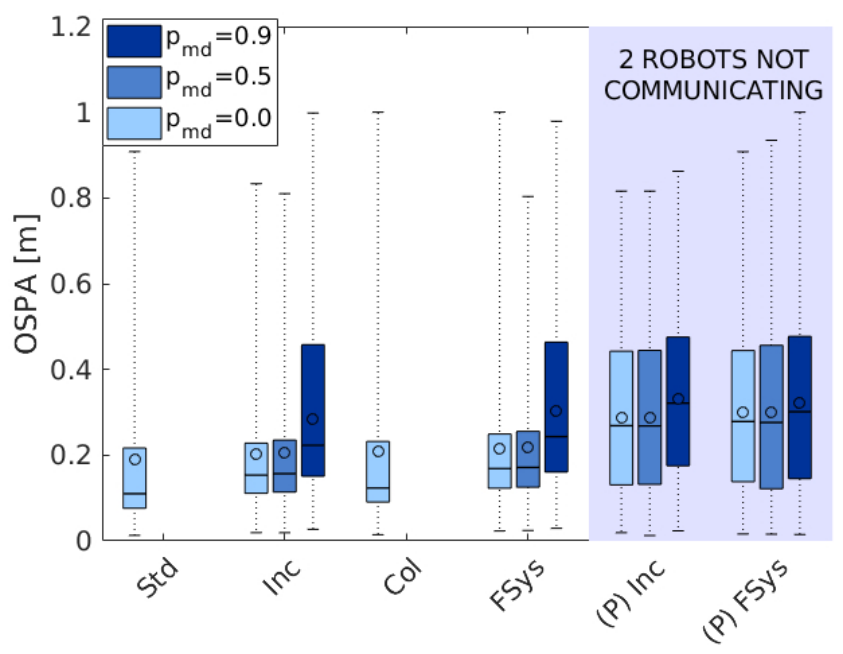

Fig. 6: Scenario I. The OSPA metric. Note that the Std and Col do not use the communicated data. Std stands for the standard GM-PHD filter; Inc stands for FI-GM-PHD filter with inception step only; Col stands for FI-GM-PHD filter with the coalition step only; FSys stands for the full FI-GMPHD system; $(P)$ stands for a situation, when a subset of robots is not communicating.

formation) and maintenance of a diamond formation. As a baseline for comparison, we use an experiment where robots rely on i) only communication, but where robot roles are given a priori, ii) only communication with dynamic role assignment, so that the robots take optimal paths to the formation. We study the performance of iii) the standard GM-PHD filter, iv) the inception step only, v) the coalition step only, and vi) the full FI-GM-PHD system. The methods (i-ii, iv and vi) are tested with message drop probabilities $p_{m d}=0.0$, $p_{m d}=0.5$ and $p_{m d}=0.9$ and in a situation when two robots are not communicating (and where $p_{m d}=0.0$ ). For each of the ten worlds we allow maximally three trials for testing whether a method can complete successfully.

\subsection{Scenario III: Limitations}

Limitations of our methods are studied with regard to two aspects, A) challenging environments with obstacles and B) precision of robot detection.

Scenario III-A: Challenging Environments The leader robot guides a diamond-shaped formation of four robots in an environment populated with obstacles of various shapes and sizes. We use experiment with i) perfect communication with dynamic role assignment as a baseline for comparison. We study the performance of ii) the standard GM-PHD filter and iii) the full FI-GM-PHD system. In iii), only three robots communicate. For each experiment, 5 sequential runs of approximately $400 s$ are performed.

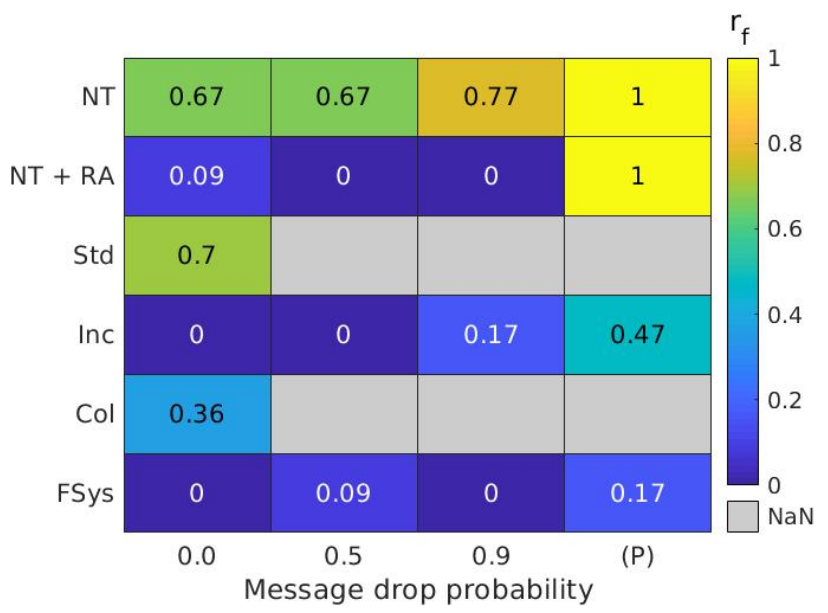

Fig. 7: Scenario II. Failure rate $r_{f}$. $N T$ stands for experiment where robots do not perform tracking, but use only communicated data; $R A$ stands for experiment where dynamic role assignment is used (indicated only for $N T$ ).

Scenario III-B: Measurement Error Three robots move on a circular trajectory in a triangle-shaped formation. We compare i) perfect communication with dynamic role assignment, ii) the standard GM-PHD filter and iii) the full FIGM-PHD system. For ii-iii, we add a random uniform error to the original measurement, with the magnitude of a) $\left.\left.\left.e_{M}=0.0 \mathrm{~m}, \mathrm{~b}\right) e_{M}=0.3 \mathrm{~m}, \mathrm{c}\right) e_{M}=0.6 \mathrm{~m}, \mathrm{~d}\right) e_{M}=1.0 \mathrm{~m}$ and e) $e_{M}=1.5 \mathrm{~m}$. For iii, only the leader robot communicates. We perform 10 sequential runs of around $120 \mathrm{~s}$.

\subsection{Scenario IV: Final Demonstration}

The goal of the final experiments is to showcase the nominal situation for which our methods are targeted, namely when the communications has temporary problems that can be overcome by taking advantage of the tracking. Four robot move on a circular trajectory. At $t_{1}=10 \mathrm{~s}$, one of the robots loses communication for $20 \mathrm{~s}$. At $t_{2}=50 \mathrm{~s}$, there is no communication between any pair of robots, for a period of $10 \mathrm{~s}$. Finally, at $t_{3}=110 \mathrm{~s}$, one robot loses communication for $10 \mathrm{~s}$. The total length of the run is $120 \mathrm{~s}$, and we perform 10 sequential runs. A i) perfect communication with dynamic role assignment is the baseline for comparison. We study the performance of ii) the standard GM-PHD filter and iii) the full FI-GM-PHD system.

\section{Results}

In the following section we use the following acronyms for labeling the methods. Std stands for the standard GM-PHD filter; Inc stands for FI-GM-PHD filter with inception step only; Col stands for FI-GM-PHD filter with the coalition 
Table 1: Scenario II: OSPA metrics

\begin{tabular}{lcccccc}
\hline & Std & Col & \multicolumn{4}{c}{ FSys } \\
$p_{m d}$ & & & 0.0 & 0.5 & 0.9 & $(\mathrm{P})$ \\
\hline OSPA mean & 0.4 & 0.4 & 0.20 & 0.22 & 0.26 & 0.32 \\
OSPA std & 0.07 & 0.3 & 0.05 & 0.05 & 0.10 & 0.03 \\
\hline
\end{tabular}

step only; FSys stands for the full FI-GM-PHD system; NT stands for experiment where robots do not perform tracking, but use only communicated data; $R A$ stands for experiment where dynamic role assignment is used (indicated only for $N T) ;(P)$ stands for a situation when a subset of robots is not communicating.

\subsection{Scenario I: Multi-Robot Tracking}

Figure 6 shows the average OSPA results calculated between the ground truth and the estimates of the five robots. The results suggest that the standard GM-PHD filter and the Col method achieve the best performance, irrespectively of whether the other methods use additional data. This is counterintuitive, as one might expect that when communicated self-localization information is added to the tracking data, this additional source of information should improve the estimates. Since the poses shared by the robots and used in the methods Inc and FSys are the self-localization poses, this data inherently incorporates the self-localization error of the tracked robots (in addition to the self-localization error of the tracking robot), so the above methods naturally perform worse than the methods that do not incorporate the error. However, even though according to the ground truth the tracking data in methods Inc and FSys appears to be inferior when communication is sparse (with high message drop probability and when not all the robots communicate), in reality the robots perform formation control based on their own self-localization data and have no access to ground truth, neither of their own nor of the neighboring robots. Therefore, formation control based on estimates that include the self-localization data in practice does not perform worse than if it relied purely on tracking. Moreover in Scenario I the robots do not rely on the tracking data for navigation, but only passively collect it. The FI-GM-PHD filter has been designed to be used for active use in formation control, where collective motion control and tracking have mutual influence on each other. It the ensuing experiments it will be shown that the proposed approach exceeds the performance of the original method in its designated applications.

\subsection{Scenario II: Initialization}

Figure 7 shows the failure rates $r_{f}$ for each of the tested methods. An experimental run is labeled as failed if the

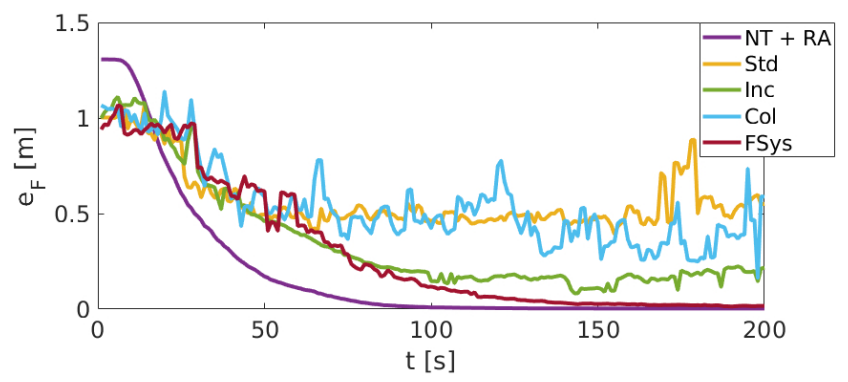

Fig. 8: Scenario II. Formation error.

robots are not capable of converging to a stable formation state, caused by the lack of data or collisions. We consider a formation to be stable when the formation error falls below a pre-established threshold and attains a steady state. If the robots are not capable of converging to a formation after initialization, or if the formation breaks after convergence without external disturbance, the formation is considered unstable. It is clear that when robots do not use tracking $(N T, N T+R A)$ and some of the robots are not communicating $(P)$, the robots always fail, hence $r_{f}=1.0$. The lack of dynamic role assignment in NT leads to frequent collisions, as the robots must shuffle when moving to designated places in the formation. In general, methods Inc and FSys perform at least as well as the baseline NT, but they also allow for formation initialization even when not all the robots communicate $(P)$. Additionally, using the formation prior in FSys leads to higher change of success than not using it in Inc. Using the formation prior but no communication in $\mathrm{Col}$ leads to slightly worse performance than FSys, with $r_{f}=0.36$, however $\mathrm{Col}$ still significantly outperforms the standard GM-PHD filter (Std) in terms of its success rate.

The tracking performance, summarized in Table 1 shows that communications always improves tracking in the FIGM-PHD method (FSys) as compared to when communications is not included (Std and $\mathrm{Col}$ ). The formation convergence as well as the precision of the formation can be deduced from Figure 8, which shows the average time-wise evolution of the formation error $e_{F}$ for $p_{m d}=0$. The convergence rate is slower for the tracking methods (Std, Inc, Col and FSys) than for the baseline NT methods. Among the tracking methods, the steady state with $e_{F} \sim 0$ is only achieved by the full FI-GM-PHD (FSys), while lack of communication (Std and $\mathrm{Col}$ ) leads to slight deformation of the final formation shape.

\subsection{Scenario III: Limitations}

Scenario III-A: Challenging Environments The trajectories of the robots using the FI-GM-PHD tracker in challenging environment can be seen in Figure 9. Even though a comparison between the FI-GM-PHD filter and the GM-PHD filter 


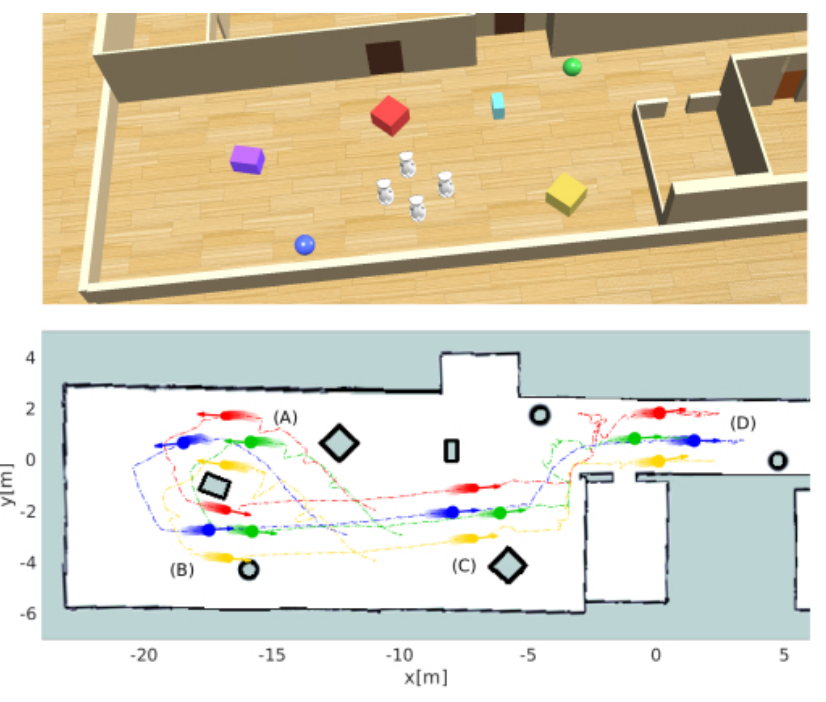

Fig. 9: Scenario III-A. Simulation screenshot (top). Trajectories of the robots at $t=62 \mathrm{~s}(\mathrm{~A}), t=114 \mathrm{~s}(\mathrm{~B}), t=190 \mathrm{~s}$ (C) and $t=270 \mathrm{~s}$ (D).

was not the aim of the experiment, the tests performed with the standard GM-PHD filter give a better insight as of why a tracking-only method $(S t d)$ is not sufficient for robust navigation of multi-robot formation in realistic environments. Figures 10 and 11 show that for the GM-PHD filter (Std) the $e_{F}$ and OSPA metrics on average keep rising until the point when the formation breaks apart at around $t=80 \mathrm{~s}$ (caused by the robots losing track of the leader or drifting apart form each other until they move out of field of view). This is not the case for the FI-GM-PHD (FSys) method, which enables periodic correction of the tracker with the communications data, even when only a subset of robots communicates. Note, that presence of obstacles that are close to the path of the robots forces deformation of the formation shape, which in turn leads to lowering the weights of the filter components that stem from the formation prior, so the coalition step of the FI-GM-PHD has a small effect on improving the tracking performance. Therefore in challenging environments the FI-GM-PHD filter with no communications is expected to perform very similarly to the standard GM-PHD filter.

Scenario III-B: Measurement Error Results summarized in Table 2 indicate, that even though larger measurement error has a negative impact on the performance of the FI-GMPHD filter, the method is still capable of providing good enough estimates to sustain the robot formation even with the measurement error of up to 1 meter. Precision of the formation, expressed by the formation error $e_{F}$, deteriorates steadily with the increase of the measurement error $e_{M}$, however it remains within reasonable bounds of less than half of robot radius. There is a similar trend in the tracking performance. For $e_{M}=1 m$ the robots, however, begin to have a

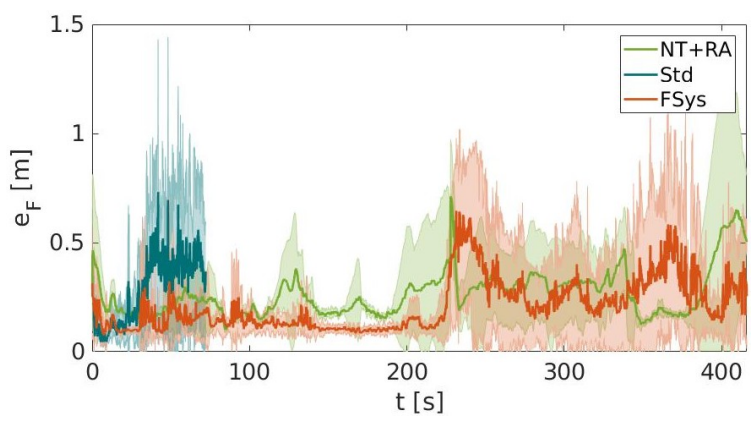

Fig. 10: Scenario III-A. Formation error.

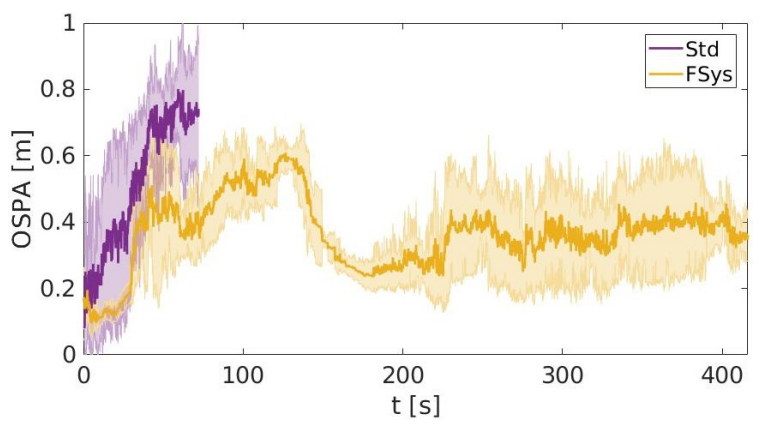

Fig. 11: Scenario III-A. OSPA.

large offset from the desired places in the formation, so the likelihood of collision increases, up to the point where for $e_{M}>1 \mathrm{~m}$ the formation is no longer guaranteed. One can also notice that even though the values of OSPA in Table 2, in the range $\sim(0.7-0.8)$ are significantly larger than in Scenarios I to III-A, the robots are still capable of maintaining the formation based on the estimates.

\subsection{Scenario IV: Final Demonstration}

Similarly as in Scenario III-B, the GM-PHD tracker alone is not capable of providing robots state information reliable enough for maintaining a formation for extended amount of time. Even in an environment free of obstacles, rotation of the formation and the associated temporary loss of neighbor tracks causes divergence in the local robot perception of the optimal assignment, which when uncorrected, leads to instability of the formation (see Figures 12 and 13, $t=42 s$ ). The performance of the FI-GM-PHD method (FSys) remains stable even when one robot is disconnected and when none of the robots communicates. The tracking error, shown in Figure 13, stays around a constant value, irrespectively of the communications status. The formation error in Figure 12 is comparable to the baseline error of formations that rely on perfect communication $(N T+R A)$. Slightly divergent behavior between $N T+R A$ and $F$ Sys at times $t=80-120 \mathrm{~s}$ occurs due to the fact that during this part of the experiment the robots are navigating closely to a wall. The $N T+R A$ method 
Table 2: Scenario III-B: Metrics

\begin{tabular}{lcccccc}
\hline & NT+RA & \multicolumn{5}{c}{ FSys } \\
$e_{M}[m]$ & & 0.0 & 0.3 & 0.6 & 0.75 & 1.0 \\
\hline$e_{F}$ mean & 0.2 & 0.27 & 0.32 & 0.36 & 0.32 & 0.34 \\
$e_{F}$ std & 0.03 & 0.1 & 0.1 & 0.1 & 0.16 & 0.1 \\
\hline OSPA mean & - & 0.7 & 0.67 & 0.76 & 0.77 & 0.83 \\
OSPA std & - & 0.04 & 0.04 & 0.05 & 0.07 & 0.05 \\
\hline
\end{tabular}

leads to higher temporary formation distortion, while the FSys method results in the formation staying closer to the desired shape, but taking longer to recover.

\subsection{Discussion}

Highly dynamic scenarios with multiple robots moving close-by in a coordinated fashion are decidedly challenging for tracking. While the multi-target tracking methods work well with well-separated targets and reliable measurements (Vo and Ma 2006), long-term occlusions, convoluted tracks, and sensor-induced clutter hardly distinguishable from the real tracks cause deterioration of tracking performance up to a point, where the reliability of estimates is insufficient for maintaining a formation. Structured indoor environments, where the robots must navigate along complex paths and around obstacles increase the difficulty of the problem even further.

Furthermore, in the particular situation, when the estimates must be matched with the formation roles, and when the role assignment is necessary, any tracking errors are escalated. If the assignment is inconsistent among the robots, the different perception of the overall formation state may not allow the consensus methods to converge. For this reason only a subset of robots can maintain formation with tracking-only data for longer periods of time. With a small numbers of robots, the complexity of association and probability of wrong assignment is low, therefore larger proportion of non-communicating robots is allowed. With increasing numbers of robots, this proportion becomes smaller. Therefore for groups of robots larger than the size demonstrated in this paper, one should consider anonymous (IDless) formations (e.g. potential fields), where each robot keeps a constant distance from each estimated neighbor irrespective of the identity of that robot. Such methods are much less prone to tracking errors, at the expense of the precision of the formation shape.

Incorporating communicated data in the tracking filter can provide reliable data used for reinforcing the existing targets or for adding targets that cannot be tracked using other means. In the conference version of this paper (Wasik et al 2017a) the communicated data is included as intensity in the prediction step (analogously to the birth inten-

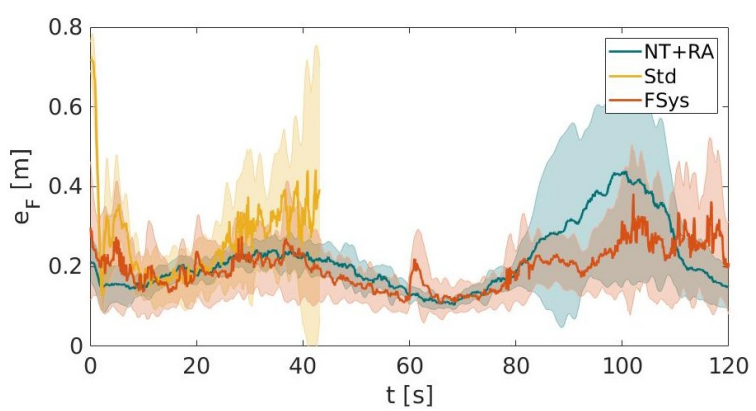

Fig. 12: Scenario IV. Formation error.

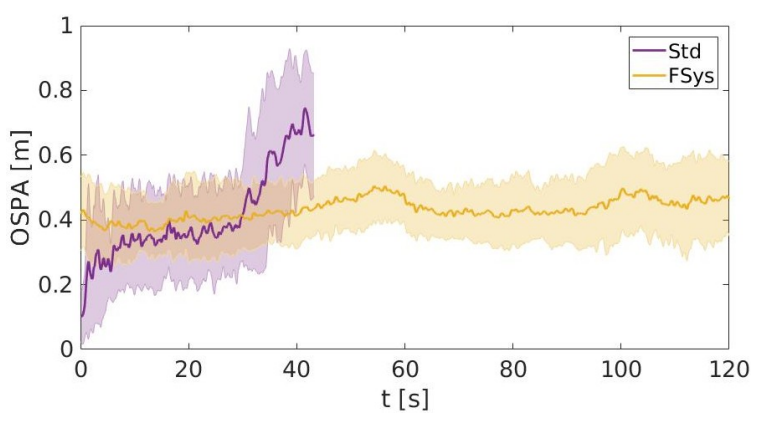

Fig. 13: Scenario IV. OSPA.

sity), while in this work the communicated data is integrated as measurements in the update step. In preparation for this publication we compared the two approaches in a series of tests (they are not included in the final version of the manuscript), the results of which show that the second method, where communicated data is used as measurements, systematically yields moderately better performance. More importantly, this method is simple and intuitive. It does not require complex parametrization that depends on the experimental setup, as it is the case with the first method. Irrespective of the method, when adding communicated data in the filter it is important to adapt the selection step of the PHD filter to discourage double-counting of the targets and prevent inconsistencies in the targets weights.

Finally, incorporating formation geometry in the tracker might lead to worse performance than if such information was not used. While the posterior enforcement stage of the coalition step (step 1-4 in Algorithm 2) is robust to challenging scenarios, and in the worst case it does not improve performance of tracking, the novelty stage (step 6 in Algorithm 2) can create a virtual robot in place of a robot that singles out from the formation. This can happen when all the robots except one are close to desired places in the formation, and is common with leader-follower formations, when the leader moves too fast for the formation to converge. One possible solution to this problem is to reinforce the followerleader edges with higher weights. However, in some environments characterized by high clutter, where the followers 
might get stuck behind an obstacle, one might have to consider disabling the novelty step. Using formation geometry is particularly advantageous in situations where a subset of robots cannot communicate and measurements are unreliable, but the formation is capable of physically maintaining a close-to-desired formation. In such instances, the coalition step can determine whether the formation continues to function or if it fails.

\section{Conclusions and Future Work}

The paper presented a strategy for providing reliable and robust robot state estimates to be used for formation control. The aim of our work was to build a backup system for situations, when the primary source of information, namely communication, is of low rate or when it fails. For safety reasons, such backup system is necessary for establishing cooperative multi-robot navigation in human-populated environments, which is the long-term goal of this work. Our method combined absolute positions exchanged by the robots, information about the formation geometry and sensory detections in an extension of the GM-PHD filter. The experiments showed that our approach, the FI-GM-PHD method, is capable of maintaining the state estimates even when longduration occlusions occur, and improves awareness of the situation when the communications is sporadic or suffers from short-term outage. Moreover, the results showed that the proposed tracking strategy allows for sustaining formations in cluttered environments, with high measurement uncertainty and low quality of communication. We studied the limitations of the method, focusing on the performance in situations, where spatial configuration of the robot is far off from the desired formation geometry, including initialization and cluttered environments. The proposed system not only outperformed the standard tracking, but also proved comparable to situation with perfect communications.

In future work, we will test the scalability of the method with larger number of simulated robots. We plan to better analyze the sensor model, to reduce the measurement error and amount of clutter stemming from sensor imperfections. The FI-GM-PHD method will be validated with real robots and tested in an indoor arena populated with obstacles.

\section{Additional Material}

Videos accompanying this paper can be found at: https://disal.epfl.ch/research/ InstitutionalRoboticsFormations.

\section{References}

Adams M, Vo BN, Mahler R, Mullane J (2014) Slam gets a phd: New concepts in map estimation. IEEE Robotics Automation Magazine 21(2):26-37
Ahmad A, Lawless G, Lima P (2017) An online scalable approach to unified multirobot cooperative localization and object tracking. IEEE Transactions on Robotics 33(5):1184-1199

Alonso-Mora J, Knepper R, Siegwart R, Rus D (2015) Local motion planning for collaborative multi-robot manipulation of deformable objects. In: 2015 IEEE Int. Conf. on Robotics and Automation, pp 5495-5502

Alur R, Das A, Esposito J, Fierro R, Grudic G, Hur Y, Kumar V, Lee I, Ostrowski JP, Pappas G, Southall B, Spletzer J, Taylor CJ (2001) A Framework and Architecture for Multirobot Coordination, Springer Berlin Heidelberg, Berlin, Heidelberg, pp 303-312

Bar-Shalom Y (1990) Multitarget-multisensor tracking: advanced applications. Norwood, MA, Artech House, 1990, 391 p 1

Bar-Shalom Y, Fortmann T (1988) Tracking and Data Association. Mathematics in Science and Engineering Series, Academic Press

Bishop AN (2011) A very relaxed control law for bearing-only triangular formation control. IFAC Proc Volumes 44(1):5991 - 5998

Blackman S, Popoli R (1999) Design and Analysis of Modern Tracking Systems. Artech House radar library, Artech House

Cao M, Yu C, Anderson BD (2011) Formation control using rangeonly measurements. Automatica 47(4):776 - 781

Dames P (2017) Distributed multi-target search and tracking using the phd filter. In: 2017 Int. Symposium on Multi-Robot and MultiAgent Systems, pp 1-8

Dames P, Kumar V (2013) Cooperative multi-target localization with noisy sensors. In: 2013 IEEE Int. Conf. on Robotics and Automation, pp 1877-1883

Dias D, Lima PU, Martinoli A (2016) Distributed formation control of quadrotors under limited sensor field of view. In: Proc. of the 15th Int. Conf. on Autonomous Agents and Multiagent Systems, ACM, pp 1087-1095

Ester M, Kriegel HP, Sander J, Xu X (1996) A density-based algorithm for discovering clusters in large spatial databases with noise. In: Proc. of the Second Int. Conf. on Knowledge Discovery and Data Mining, AAAI Press, pp 226-231

Falconi R, Gowal S, Martinoli A (2010) Graph-based distributed control of non-holonomic vehicles endowed with local positioning information engaged in escorting missions. In: IEEE Int. Conf. on Robotics and Automation, pp 3207-3214

Franchi A, Oriolo G, Stegagno P (2013) Mutual localization in multirobot systems using anonymous relative measurements. The Int Journal of Robotics Research 32(11):1302-1322

Fredslund J, Matarić MJ (2002) A general algorithm for robot formations using local sensing and minimal communication. IEEE Trans on Robotics and Automation 18(5):837-846

Han Z, Guo K, Xie L, Lin Z (2018) Integrated relative localization leader-follower formation control. IEEE Transactions on Automatic Control pp 1-1

Jiang B, Deghat M, Anderson BDO (2017) Simultaneous velocity and position estimation via distance-only measurements with application to multi-agent system control. IEEE Transactions on Automatic Control 62(2):869-875

Kuhn HW (1955) The hungarian method for the assignment problem. Naval Research Logistics Quarterly 2(1-2):83-97

Lewis MA, Tan KH (1997) High precision formation control of mobile robots using virtual structures. Autonomous Robots 4(4):387-403

Mahler RP (2003) Multitarget bayes filtering via first-order multitarget moments. IEEE Transactions on Aerospace and Electronic systems 39(4):1152-1178

Melo J, Matos A (2014) A phd filter for tracking multiple auvs. In: 2014 Oceans - St. John's, pp 1-8

Michael N, Zavlanos MM, Kumar V, Pappas GJ (2008) Distributed multi-robot task assignment and formation control. In: IEEE Int. Conf. on Robotics and Automation, pp 128-133

Michel O (2004) Cyberbotics ltd. webots: professional mobile robot simulation. Int Journal of Advanced Robotic Systems 1(1):5 
Monteiro S, Bicho E (2010) Attractor dynamics approach to formation control: theory and application. Autonomous Robots 29(3):331355

Moratuwage D, Vo BN, Wang D (2012) A hierarchical approach to the multi-vehicle slam problem. In: 2012 15th Int. Conf. on Information Fusion, pp 1119-1125

Nägeli T, Meier L, Domahidi A, Alonso-Mora J, Hilliges O (2017) Real-time planning for automated multi-view drone cinematography. ACM Trans Graph 36(4):132:1-132:10

Oh S, Russell S, Sastry S (2009) Markov chain monte carlo data association for multi-target tracking. IEEE Transactions on Automatic Control 54(3):481-497

Olfati-Saber R, Fax JA, Murray RM (2007) Consensus and cooperation in networked multi-agent systems. Proc of the IEEE 95(1):215233

Panta K, Clark DE, Vo BN (2009) Data association and track management for the gaussian mixture probability hypothesis density filter. IEEE Transactions on Aerospace and Electronic Systems 45(3):1003-1016

Qiu C, Zhang Z, Lu H, Luo H (2015) A survey of motion-based multitarget tracking methods. Progress In Electromagnetics Research B 62:195-223

Saldaña D, Assunção R, Hsieh MA, Campos MFM, Kumar V (2017) Cooperative prediction of time-varying boundaries with a team of robots. In: 2017 Int. Symposium on Multi-Robot and Multi-Agent Systems, pp 9-16

Sapkota KR, Roelofsen S, Rozantsev A, Lepetit V, Gillet D, Fua P, Martinoli A (2016) Vision-based unmanned aerial vehicle detection and tracking for sense and avoid systems. In: 2016 IEEE/RSJ Int. Conf. on Intelligent Robots and Systems, pp 1556-1561

Saska M, Krtk V, Spurn V, Ba T (2017) Documentation of dark areas of large historical buildings by a formation of unmanned aerial vehicles using model predictive control. In: 2017 22nd IEEE Int. Conf. on Emerging Technologies and Factory Automation, pp 1-8

Schuhmacher D, Vo BT, Vo BN (2008) A consistent metric for performance evaluation of multi-object filters. IEEE Transactions on Signal Processing 56(8):3447-3457

Soares JM, Aguiar AP, Pascoal AM, Martinoli A (2013) Joint ASV/AUV range-based formation control: theory and experimental results. In: IEEE Int. Conf. on Robotics and Automation, pp 5579-5585

Stegagno P, Cognetti M, Rosa L, Peliti P, Oriolo G (2013) Relative localization and identification in a heterogeneous multi-robot system. In: 2013 IEEE Int. Conf. on Robotics and Automation, pp 1857-1864

Streit RL, Luginbuhl TE (1994) Maximum likelihood method for probabilistic multihypothesis tracking. In: SPIE's Int. Symposium on Optical Engineering and Photonics in Aerospace Sensing, pp 394405

Sun Z, Xia Y (2014) Consensus-based formation control with dynamic role assignment. In: The 26th Chinese Control and Decision Conf., IEEE, pp 3681-3686

Vasic M, Martinoli A (2015) A collaborative sensor fusion algorithm for multi-object tracking using a gaussian mixture probability hypothesis density filter. Proc of the 2015 IEEE 18th Int Conf on Intelligent Transportation Systems pp 491-498

Ventura R, Ahmad A (2015) Towards optimal robot navigation in urban homes. In: RoboCup 2014: Robot World Cup XVIII, Springer, Cham, Switzerland, Lecture Notes in Computer Science ; 8992, pp 318-331

Vo BN, Ma WK (2006) The gaussian mixture probability hypothesis density filter. IEEE Transactions on signal processing 54(11):4091-4104

Wasik A, Pereira JN, Ventura R, Lima PU, Martinoli A (2016a) Graphbased distributed control for adaptive multi-robot patrolling using local formation transformation. In: IEEE Int. Conf. on Intelligent
Robots and Systems, pp 1721-1728

Wasik A, Ventura R, Pereira JN, Lima PU, Martinoli A (2016b) Lidarbased relative position estimation and tracking for multi-robot systems. In: Robot 2015: Second Iberian Robotics Conf., Springer Int. Publishing, Cham, pp 3-16

Wasik A, Martinoli A, Lima PU (2017a) A robust relative positioning system for multi-robot formations leveraging an extended gm-phd filter. Proc of the First Int Symposium on Multi-Robot and MultiAgent Systems pp 7. 71-77

Wasik AB, Martinoli A, Lima PU (2017b) An institutional robotics approach to the design of socially aware multi-robot behaviors. Proc of the RO-MAN 2017 Workshop on Towards Intelligent Social Robots: Social Cognitive Systems in Smart Environments pp 6. 2-7

Ye M, Anderson BDO, Yu C (2017) Bearing-only measurement self-localization, velocity consensus and formation control. IEEE Transactions on Aerospace and Electronic Systems 53(2):575-586

Zavlanos MM, Pappas GJ (2008) Dynamic assignment in distributed motion planning with local coordination. IEEE Trans on Robotics 24(1):232-242

Zhang Y, Song G, Qiao G, Li Z, Li Y, Song A (2013) Strategy research of role assignment and formation control for multi-robot systems. In: 2013 IEEE Int. Conf. on Robotics and Biomimetics, pp 958963 